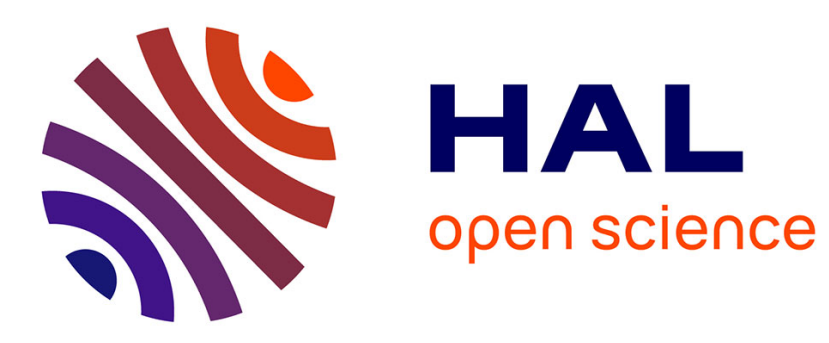

\title{
Bootstrap for almost cyclostationary processes with jitter effect
}

\author{
Dominique Dehay, Anna Dudek, Mohamed El Badaoui
}

\section{To cite this version:}

Dominique Dehay, Anna Dudek, Mohamed El Badaoui. Bootstrap for almost cyclostationary processes with jitter effect. Digital Signal Processing, 2018, 73, pp.93-105. 10.1016/j.dsp.2017.11.002 . hal$01430394 \mathrm{v} 3$

\section{HAL Id: hal-01430394 \\ https://hal.science/hal-01430394v3}

Submitted on 5 Feb 2018

HAL is a multi-disciplinary open access archive for the deposit and dissemination of scientific research documents, whether they are published or not. The documents may come from teaching and research institutions in France or abroad, or from public or private research centers.
L'archive ouverte pluridisciplinaire $\mathbf{H A L}$, est destinée au dépôt et à la diffusion de documents scientifiques de niveau recherche, publiés ou non, émanant des établissements d'enseignement et de recherche français ou étrangers, des laboratoires publics ou privés. 


\title{
Bootstrap for almost cyclostationary processes with jitter effect
}

\author{
Dominique Dehay $^{a}$, Anna E. Dudek ${ }^{a, b}$, Mohamed El Badaoui $^{c, d}$
}

${ }^{a}$ Institut de Recherche Mathématique de Rennes, UMR CNRS 6625, Université Rennes 2, France.

${ }^{b}$ AGH University of Science and Technology, al. Mickiewicza 30, 30-059 Krakow, Poland. email: aedudek@agh.edu.pl; tel. +486174405; fax: +486334199.

${ }^{c}$ University of Lyon, UJM-St-Etienne, LASPI, EA3059, F-42023, Saint-Etienne, France.

${ }^{d}$ Safran Tech, Rue des Jeunes Bois - Châteaufort 78772 Magny-les-Hameaux, France.

\begin{abstract}
In this paper we consider almost cyclostationary processes with jitter effect. We propose a bootstrap approach based on the Moving Block Bootstrap method to construct pointwise and simultaneous confidence intervals for the Fourier coefficients of the autocovariance function of such processes. In the simulation study we showed how our results can be used to detect the significant frequencies of the autocovariance function. We compared the behavior of our approach for jitter effects caused by perturbations from two distributions, namely uniform and truncated normal. Moreover, we present a real data application of our methodology.
\end{abstract}

Keywords: almost cyclostationarity, bootstrap, jitter, significance frequency detection.

\section{Introduction}

In this paper we consider almost cyclostationary (ACS) processes. They are generalizations of cyclostationary (CS) processes. A process $X(t)$ with finite second moments is called CS with period $d$, if it has periodic mean and covariance function, i.e.,

$$
\mathrm{E}(X(t+d))=\mathrm{E}(X(t)) \quad \text { and } \quad \operatorname{Cov}(X(t), X(s))=\operatorname{Cov}(X(t+d), X(s+d)) .
$$

For more details we refer the reader to [1].

Moreover, a process $X(t)$ with finite second moments is called ACS, if its mean and autocovariance functions are almost periodic. Let us recall that almost periodic functions were introduced by Besicovitch in [2]. A function $f: \mathcal{R} \rightarrow \mathcal{R}$ is called almost periodic if for every $\varepsilon>0$ there exists a number $l_{\varepsilon}$ such that for any interval of length greater than $l_{\varepsilon}$, there is a number $p_{\varepsilon}$ in this interval such that

$$
\sup _{t \in \mathcal{R}}\left|f\left(t+p_{\varepsilon}\right)-f(t)\right|<\varepsilon .
$$

Equivalently, the almost periodic functions can be defined as the uniform limits of trigonometric polynomials (see [2]). For more information on ACS processes we refer the reader to [3].

To analyze ACS processes, Fourier analysis is often applied. Fourier expansions of the mean and the autocovariance function are used to detect significant frequencies. Although, results establishing the estimators of the Fourier coefficients and their properties are well known (see [4]), in practical applications 
one needs also a method to obtain the range of possible values of the considered parameters. Unfortunately, the asymptotic confidence intervals cannot be constructed because the asymptotic variances of the estimators depend on the unknown parameters. Thus, to compute confidence intervals resampling methods are used. They allow us to approximate the distribution of the statistics of interest.

One of the most popular resampling techniques is the bootstrap method. It was introduced by Efron in [5]. The method was initially designed for independent and identically distributed data, but in the late 1980s and beginning of the 1990s, there appeared modifications dedicated for stationary time series (see [6] and [7]). Finally, techniques for nonstationary processes have been developed in the last 10 years. Methods dedicated to stationary or nonstationary time series are designed to preserve the dependence structure contained in the data. The idea is to randomly sample blocks of observations and hence to keep inside of each block the dependence structure contained in the original data. Currently there exist three block bootstrap methods that can be applied to CS/ACS processes. These are the Moving Block Bootstrap (MBB) introduced independently in [6] and [7], the Generalized Seasonal Block Bootstrap (GSBB) proposed in [8], and the Generalized Seasonal Tapered Block Bootstrap (GSTBB) proposed in [9]. All can be used for CS processes, but since the GSBB and the GSTBB require knowledge of the period length, they cannot be applied to the ACS case. The first bootstrap consistency result for CS/ACS processes was obtained in 2007 by Synowiecki in [10]. The author showed validity of the MBB for the overall mean of the ACS time series. Dudek et al. proved the GSBB consistency for the overall mean and the seasonal means in [8]. The corresponding results for the GSTBB can be found in [9]. The applicability of the modified MBB for the Fourier coefficient of the mean and the autocovariance functions of ACS time series was proved in [11]. Fourier coefficient of the mean and the autocovariance functions of CS time series were considered in [12] (GSBB) and [9] (GSTBB). Moreover, Dehay and Dudek showed the MBB validity for Fourier coefficient of the mean and the autocovariance functions of ACS continues time process that is not fully observed (see [13] and [14]).

In the following we extend applicability of the bootstrap method to the ACS processes, which are observed in instants that are randomly disturbed. This effect is called jitter. It appears in many signal analysis problems ([15], [16], [17]), e.g., receiver design in telecommunication, audio applications, optical encoders, etc. Jitters in clock signals are typically caused by noise or other disturbances in the system. Contributing factors include thermal noise, power supply variations, loading conditions, device noise, and interference coupled from nearby circuits.

When acquiring a signal, the jitter of the sampling clock can be voluntary or involuntary. For example, in the case of compressed sensing it is essential to achieve a random signal acquisition. In practice this operation can be performed by adding a random jitter on the clock signal. In other applications, the jitter can be undergoing. This is the case for the angular acquisitions of vibratory signals issued from rotating machinery. In this situation, angular sampling is sensitive to hardware imperfections (optical encoder precision, electrical perturbation, etc.) (see [18]). The angular sampled signal quantification step or sampling frequency determination is not identical to the time domain ([19], [20]). Angular sampling is also not adapted to study time domain signals like impulse response. Some of the imperfections can be viewed as non-uniform sampling or random jitter. In these voluntary or involuntary circumstances, it appears appropriate to develop CS signal analysis tools sampled in the presence of jitter.

The paper is organized as follows. In Section 2 the problem is formulated and the considered assumptions are presented. In addition, the estimators of the Fourier coefficients are introduced and their asymptotic properties are discussed. Section 3 is dedicated to the bootstrap method. The MBB approach adapted to our problem is presented and its consistency for the Fourier coefficients of the autocovariance function is shown. Finally, the construction of the bootstrap pointwise and the simultaneous confidence intervals are 
provided. Section 4 is devoted to the alternative bootstrap technique that can be used in the considered problem. In Section 5, a simulation study is presented in which the performance of the proposed bootstrap method is verified. Finally, in Section 6 the real data vibratory gear vibration signal is analyzed and the obtained results are discussed in Section 7.

\section{Problem formulation}

Let $X=\{X(t), t \in \mathbb{R}\}$ be a zero-mean real-valued process that is uniformly almost cyclostationary (UACS), i.e., for any $s \in \mathbb{R} \mathrm{E}\left(X^{2}(s)\right)<\infty$ and autocovariance function $B(t, \tau)=\operatorname{Cov}\left(X_{t}, X_{t+\tau}\right)=$ $\mathrm{E}(X(t) X(t+\tau))$ is almost periodic in $t$ uniformly in $\tau$.

In the following, we use notation introduced in [21].

The process $X(t)$ is not observed continuously but only in instants $t_{k}=k h+U_{k}, h>0$. Hence one observes the discrete time process $X_{k}=\left\{X\left(k h+U_{k}\right), k \in \mathcal{Z}\right\}$. Random variables $U_{k}$ are independent and identically distributed (iid) and are independent of $X$. They can be considered as random errors. In fact we assume that each instant in which the process is observed is disturbed. In the sequel we assume that $h>0$ is fixed and small enough to avoid aliasing. In [18] a similar model was considered with $U_{k}$ being iid random variables from the standard normal distribution.

In this paper we focus on the Fourier analysis of $X$. Generally, for a fully observed process $X$, the Fourier coefficients of the autocovariance function are of the form

$$
a(\lambda, \tau)=\lim _{T \rightarrow \infty} \frac{1}{T} \int_{0}^{T} \mathrm{E}(X(t) X(t+\tau)) \exp (-i \lambda s) d s
$$

For a continuous time observation of a UACS process $X$ the estimator

$$
\widehat{a}_{T}(\lambda, \tau)=\frac{1}{T} \int_{0}^{T} X(t) X(t+\tau) \exp (-i \lambda t) d t
$$

is consistent and asymptotically normal (see, e.g., [4], [22]).

To estimate the Fourier coefficients of the autocovariance function of $X$ in our case, we need to use some approximation. The problem is caused by the fact that we need to know values $X(t) X(t+\tau)$. Since $\tau$ is not always a multiple of $h$, we approximate $\tau$ by the nearest multiple of $h$. Let $k_{\tau}$ be the nearest integer to $\tau / h$. We have

$$
\frac{\tau}{h}-\frac{1}{2}<k_{\tau}<\frac{\tau}{h}+\frac{1}{2}
$$

Finally, $U_{k, \tau}=U_{k+k_{\tau}}+k_{\tau} h-\tau$ is a time perturbation for the time moment $k h+\tau$.

We observe a sample $\left\{X\left(k h+U_{k}\right): 1 \leq k \leq n\right\}$. The estimator of $a(\lambda, \tau)$ is defined as follows

$$
\widetilde{a}(\lambda, \tau)=\frac{1}{n} \sum_{k=1}^{n} \widetilde{b}_{k}(\lambda, \tau),
$$

where

$$
\widetilde{b}_{k}(\lambda, \tau)=X\left(k h+U_{k}\right) X\left(\left(k+k_{\tau}\right) h+U_{k+k_{\tau}}\right) \exp (-i \lambda k h)
$$

and $0 \leq k \leq n$ and $0 \leq k+k_{\tau} \leq n$. Note that for fixed $h>0$, the time series $\widetilde{b}_{k}(\lambda, \tau)$ is ACS. In the next subsection we discuss the assumptions that we used to derive our results. 


\subsection{Assumptions}

In the sequel the following conditions are used:

(i) $X$ is sampled at a constant rate greater than the Nyquist rate with time step $h>0$ ( $h$ is small enough to avoid aliasing);

(ii) the random perturbations $U_{k}$ are iid from some distribution on $(-h / 2, h / 2)$;

(iii) set $\Lambda=\{\lambda \in \mathbb{R}: a(\lambda, \tau) \neq 0$ for some $\tau \in \mathbb{R}\}$ is finite;

(iv) $\sup _{t} \mathrm{E}\left\{|X(t)|^{8+2 \eta}\right\}<\infty$ for some $\eta>0$;

(v) $X$ has almost periodic fourth moments, i.e. for each $t \in \mathbb{R}, \mathrm{E}\left\{X(t)^{4}\right\}<\infty$; the function $\left(t, \tau_{1}, \tau_{2}, \tau_{3}\right) \mapsto \mathrm{E}\left\{X(t) X\left(t+\tau_{1}\right) X\left(t+\tau_{2}\right) X\left(t+\tau_{3}\right)\right\}$ is almost periodic in $t$ uniformly with respect to $\tau_{1}, \tau_{2}, \tau_{3}$ varying in $\mathbb{R}$;

(vi) $X(t)$ is $\alpha$-mixing and $\sum_{k=1}^{\infty} k \alpha_{X}^{\frac{\eta}{\eta+4}}(k)<\infty$;

Condition (ii) prevents permutation of observations caused by the jitter effect, i.e., we assume that the instants of the observations are perturbed but the order of the observations is unchanged. Assumption (iii) denotes that for each $\tau$ there is a finite number of non-zero coefficients $a(\lambda, \tau)$ or equivalently a finite number of significant frequencies $\lambda$. This condition is not necessary but allows us to simplify the presentation of the results. Finally, to obtain the asymptotic normality of $\widetilde{a}(\lambda, \tau)$, a mixing condition (vi) is needed. To be precise, process $X$ is called $\alpha$-mixing if $\alpha_{X}(k) \rightarrow 0$ as $k \rightarrow \infty$, where

$$
\alpha_{X}(u)=\sup _{t} \sup _{\substack{A \in \mathcal{F}_{X}(-\infty, t) \\ B \in \mathcal{F}_{X}(t+u, \infty)}}|P(A \cap B)-P(A) P(B)|,
$$

and $\mathcal{F}_{X}(-\infty, t)=\sigma(\{X(s): s \leq t\})$ and $\mathcal{F}_{X}(t+u, \infty)=\sigma(\{X(s): s \geq t+u\})$ are $\sigma$-fields generated by observations from the past and future, respectively. Thus, the $\alpha$-mixing coefficient $\alpha_{X}(u)$ measures the dependence between the past and the future information carried by the process $X$ when the horizon (or delay) is $u$. When $\alpha_{X}(u)=0$, it means that observations that are $u$ time unites apart are independent. The $m$-dependent time series are known to be $\alpha$-mixing. More details and examples can be found in [23].

\section{$2.2 \quad$ Properties of $\widetilde{a}(\lambda, \tau)$}

Below we state the asymptotic normality of $\widetilde{a}(\lambda, \tau)$ in the one-dimensional and in the multidimensional case. From now on, any complex number $z$ we treat as the two-dimensional vector of the form $z=$ $(\Re(z), \Im(z))^{\prime}$. By $\Re(z)$ and $\Im(z)$ we denote the real and the imaginary part of $z$. Finally, the symbol $(\cdot)^{\prime}$ denotes the transpose of a vector.

Theorem 2.1 Assume that conditions $(i)-\left(\right.$ iii) and (vi) are fulfilled. Moreover, let $\sup _{t} \mathrm{E}\left\{|X(t)|^{4+\eta}\right\}<$ $\infty$ for some $\eta>0$. Then, $\sqrt{n h}(\widetilde{a}(\lambda, \tau)-\mathrm{E}(\widetilde{a}(\lambda, \tau)))$ converges in distribution to the two-dimensional Gaussian law $N_{2}(0, B(\lambda, \tau))$, where

$$
\begin{aligned}
B(\lambda, \tau) & =\frac{1}{2} \int_{\mathbb{R}}\left(b_{c}(0, \tau, t, t+\tau) S_{1}(\lambda t)+b_{s}(0, \tau, t, t+\tau) S_{2}(\lambda t)\right. \\
& \left.+b_{c}(2 \lambda, \tau, t, t+\tau) S_{3}(\lambda t)+b_{s}(2 \lambda, \tau, t, t+\tau) S_{4}(\lambda t)\right) d t
\end{aligned}
$$


and

$$
\begin{aligned}
& b_{c}(\lambda, u, v, w)=\lim _{T \rightarrow \infty} \frac{1}{T} \int_{0}^{T} \operatorname{Cov}(X(s) X(s+u), X(s+v) X(s+w)) \cos (\lambda s) d s, \\
& b_{s}(\lambda, u, v, w)=\lim _{T \rightarrow \infty} \frac{1}{T} \int_{0}^{T} \operatorname{Cov}(X(s) X(s+u), X(s+v) X(s+w)) \sin (\lambda s) d s, \\
& S_{1}(\theta)=\left[\begin{array}{rr}
\cos (\theta) & \sin (\theta) \\
-\sin (\theta) & \cos (\theta)
\end{array}\right], \quad S_{2}(\theta)=\left[\begin{array}{rr}
\sin (\theta) & -\cos (\theta) \\
\cos (\theta) & \sin (\theta)
\end{array}\right], \\
& S_{3}(\theta)=\left[\begin{array}{rr}
\cos (\theta) & \sin (\theta) \\
\sin (\theta) & -\cos (\theta)
\end{array}\right], \quad S_{4}(\theta)=\left[\begin{array}{rr}
-\sin (\theta) & \cos (\theta) \\
\cos (\theta) & \sin (\theta)
\end{array}\right] .
\end{aligned}
$$

The result above is a direct consequence of Theorem 2.6 in [24]. Before we recall the multidimensional version of Theorem 2.1, we introduce here some additional notation. By $\boldsymbol{\lambda}$ and $\boldsymbol{\tau}$ we denote $r$-dimensional vectors of frequencies and shifts of the form

$$
\boldsymbol{\lambda}=\left(\lambda_{1}, \ldots, \lambda_{r}\right)^{\prime}, \quad \boldsymbol{\tau}=\left(\tau_{1}, \ldots, \tau_{r}\right)^{\prime} .
$$

Additionally,

$$
a(\boldsymbol{\lambda}, \boldsymbol{\tau})=\left(\Re\left(a\left(\lambda_{1}, \tau_{1}\right)\right), \Im\left(a\left(\lambda_{1}, \tau_{1}\right)\right), \ldots, \Re\left(a\left(\lambda_{r}, \tau_{r}\right)\right), \Im\left(a\left(\lambda_{r}, \tau_{r}\right)\right)\right)^{\prime}
$$

and $\widetilde{a}(\boldsymbol{\lambda}, \boldsymbol{\tau})$ is its estimator.

Theorem 2.2 Under the assumptions of Theorem 2.1, $\sqrt{n h}(\widetilde{a}(\boldsymbol{\lambda}, \boldsymbol{\tau})-\mathrm{E}(\widetilde{a}(\boldsymbol{\lambda}, \boldsymbol{\tau})))$ converges in distribution to the $2 r$-dimensional Gaussian distribution $N_{2 r}(0, B(\boldsymbol{\lambda}, \boldsymbol{\tau}))$, where elements of $B(\boldsymbol{\lambda}, \boldsymbol{\tau})$ are calculated as follows:

$$
\begin{aligned}
B\left(\lambda_{i}, \lambda_{j}, \tau_{i}, \tau_{j}\right) & =\lim _{n \rightarrow \infty} n h \operatorname{Cov}\left(\widetilde{a}\left(\lambda_{i}, \tau_{i}\right), \widetilde{a}\left(\lambda_{j}, \tau_{j}\right)\right) \\
& =\frac{1}{2} \int_{\mathbb{R}}\left(b_{c}\left(\lambda_{i}-\lambda_{j}, \tau_{i}, t, t+\tau_{j}\right) S_{1}\left(\lambda_{j} t\right)+b_{s}\left(\lambda_{i}-\lambda_{j}, \tau_{i}, t, t+\tau_{j}\right) S_{2}\left(\lambda_{j} t\right)\right. \\
& \left.+b_{c}\left(\lambda_{i}+\lambda_{j}, \tau_{i}, t, t+\tau_{j}\right) S_{3}\left(\lambda_{j} t\right)+b_{s}\left(\lambda_{i}+\lambda_{j}, \tau_{i}, t, t+\tau_{j}\right) S_{4}\left(\lambda_{j} t\right)\right) d t
\end{aligned}
$$

for $i,=1 \ldots, r$. The symbols $b_{c}(\lambda, u, v, w), b_{s}(\lambda, u, v, w)$ and matrices $S_{1}(\theta), \ldots, S_{4}(\theta)$ are defined in Theorem 2.1.

One may note that the asymptotic covariance matrix depends on unknown parameters and in practice is almost impossible to estimate. Thus, to calculate confidence intervals, we propose to apply resampling methods. In the next section we introduce a bootstrap algorithm to construct a family of estimates which will permit us to determine the empirical confidence intervals for $a(\lambda, \tau)$.

\section{Bootstrap method}

Before we present our bootstrap algorithm, we introduce here a decomposition of $\widetilde{a}(\lambda, \tau)$.

$$
\widetilde{a}(\lambda, \tau)=\frac{1}{n} \sum_{k=1}^{n} \widetilde{b}_{k}(\lambda, \tau)=\frac{1}{n} \sum_{k=1}^{n} \ddot{b}_{k}(\tau) \exp (-i \lambda k h),
$$


where

$$
\ddot{b}_{k}(\tau)=X\left(k h+U_{k}\right) X\left(\left(k+k_{\tau}\right) h+U_{k+k_{\tau}}\right) .
$$

Note that $\ddot{b}_{k}(\tau)$ is an ACS time series (see, e.g., [3]).

Let us recall that we observe a sample $\left(X\left(h+U_{1}\right), X\left(2 h+U_{2}\right), \ldots, X\left(n h+U_{n}\right)\right)$. In the following we perform bootstrap on

$$
\left(\ddot{b}_{1}(\tau), \ldots, \ddot{b}_{n}(\tau)\right)
$$

where $\tau$ is fixed. Our bootstrap method is based on the MBB algorithm introduced independently in [6] and [7]. At first we recall the usual MBB algorithm and then we discuss its modification that we used to construct a consistent bootstrap estimator of $a(\lambda, \tau)$.

\section{MBB algorithm}

Let $\left(Y_{1}, \ldots, Y_{n}\right)$ be an observed sample and $B_{i}=\left(Y_{i}, \ldots, Y_{i+b-1}\right), i=1, \ldots, n-b+1$ be a block of observations which starts with observation $Y_{i}$ and has length $b, b \in \mathbb{N}$.

1. Choose a block size $b<n$. Then our sample can be divided into $l$ blocks of length $b$ and the remaining part is of length $r$, i.e., $n=l b+r, r=0, \ldots, b-1$.

2. From the set $\left\{B_{1}, \ldots, B_{n-b+1}\right\}$ choose randomly with replacement $l+1$ blocks $B_{1}^{*}, \ldots, B_{l+1}^{*}$. The probability of choosing any block is $1 /(n-b+1)$.

3. Join the selected $l+1$ blocks $\left(B_{1}^{*}, \ldots, B_{l+1}^{*}\right)$ and take the first $n$ observations to get the bootstrap sample $\left(Y_{1}^{*}, \ldots, Y_{n}^{*}\right)$ of the same length as the original one.

To obtain the bootstrap sample in our case we will not apply the MBB to the sample (8) directly, since we would entirely lose the information about the time indices of selected observations. This information is crucial to construct the bootstrap consistent estimator of $a(\lambda, \tau)$. Thus, we use the MBB to

$$
\left(\left(\ddot{b}_{1}(\tau), 1\right), \ldots,\left(\ddot{b}_{n}(\tau), n\right)\right) \text {. }
$$

The second coordinate in each pair corresponds to the time index of the observation.

\section{Bootstrap algorithm:}

1. Choose the block length $b<n$. Then our sample can be divided into $l$ blocks of length $b$ and the remaining part is of length $r$, i.e. $n=l b+r, r=0, \ldots, b-1$.

2. For $t=1,2, \ldots, n-b+1$ let $B_{t}=\left(\left(\ddot{b}_{t}(\tau), t\right), \ldots,\left(\ddot{b}_{t+b-1}(\tau), t+b-1\right)\right)$ be a block of the length $b$. From the set $\left\{B_{1}, \ldots, B_{n-b+1}\right\}$ we choose randomly with replacement $l+1$ blocks $B_{1}^{*}, \ldots, B_{l+1}^{*}$. This means that

$$
P^{*}\left(B_{i}^{*}=B_{j}\right)=\frac{1}{n-b+1} \quad \text { for } \quad i=1, \ldots, l+1, j=1, \ldots, n-b+1,
$$

where $P^{*}$ denotes the conditional probability given the sample $\left\{X\left(k h+U_{k}\right): 1 \leq k \leq n\right\}$.

3. Join the selected $l+1$ blocks $\left(B_{1}^{*}, \ldots, B_{l+1}^{*}\right)$ and take the first $n$ observations to get the bootstrap sample $\left(\left(\ddot{b}_{1}^{*}(\tau), 1^{*}\right), \ldots,\left(\ddot{b}_{n}^{*}(\tau), n^{*}\right)\right)$ of the same length as the original one. 
The idea behind this bootstrap method was used in [13] for the Fourier coefficients of the mean function of the continuous ACS process.

We define the bootstrap estimator of $a(\lambda, \tau)$ as follows:

$$
\widetilde{a}^{*}(\lambda, \tau)=\frac{1}{n} \sum_{k=1}^{n} \ddot{b}_{k}^{*}(\tau) \exp \left(-i \lambda k^{*} h\right) .
$$

Note that using the bootstrap only for $\left(\ddot{b}_{k}(\tau), k\right)$, which does not depend on frequency $\lambda$, allows us to calculate the value of $\widetilde{a}^{*}(\lambda, \tau)$ at the same time for many frequencies $\lambda$. Bootstraping $\left(\ddot{b}_{k}(\tau) \exp (-i \lambda k h), k\right)$ requires repetition of the algorithm for each frequency. Thus, our approach leads to a substantial reduction of the computational cost.

Below we state consistency of our bootstrap approach.

Theorem 3.1 Under assumptions $(i)-(v i)$ and if $b \rightarrow \infty$ as $n \rightarrow \infty$ such that $b / n \longrightarrow 0$, we have that

$$
\rho\left(\mathcal{L}(\sqrt{n h}(\widetilde{a}(\lambda, \tau)-a(\lambda, \tau))), \mathcal{L}^{*}\left(\sqrt{n h}\left(\widetilde{a}^{*}(\lambda, \tau)-\mathrm{E}^{*} \widetilde{a}^{*}(\lambda, \tau)\right)\right)\right) \longrightarrow 0 \quad \text { in prob. },
$$

where $\rho$ is any distance metricizing convergence in distribution on $\mathbb{R}^{2}$.

By $\mathcal{L}(\sqrt{n h}(\widetilde{a}(\lambda, \tau)-a(\lambda, \tau)))$ we denote the probability law of $\sqrt{n h}(\widetilde{a}(\lambda, \tau)-a(\lambda, \tau))$ and

$\mathcal{L}^{*}\left(\sqrt{n h}\left(\widetilde{a}^{*}(\lambda, \tau)-\mathrm{E}^{*} \widetilde{a}^{*}(\lambda, \tau)\right)\right)$ is its bootstrap counterpart conditionally on the observed sample $\{X(k h+$ $\left.\left.U_{k}\right): 1 \leq k \leq n\right\}$. Moreover, $\mathrm{E}^{*}$ is the conditional expectation given the sample. The definition of convergence in probability can be found in [25].

To present the multidimensional version of Theorem 3.1 let us introduce some additional notation. Let $r, \tau \in \mathbb{N}$ be fixed and

$$
\boldsymbol{\lambda}=\left(\lambda_{1}, \ldots, \lambda_{r}\right)^{\prime},
$$

be vector of frequencies. Moreover, let

$$
\begin{aligned}
& \Re a(\boldsymbol{\lambda}, \tau)=\left(\Re a\left(\lambda_{1}, \tau\right), \ldots, \Re a\left(\lambda_{r}, \tau\right)\right)^{\prime}, \\
& \Im a(\boldsymbol{\lambda}, \tau)=\left(\Im a\left(\lambda_{1}, \tau\right), \ldots, \Im a\left(\lambda_{r}, \tau\right)\right)^{\prime},
\end{aligned}
$$

and

$$
a(\boldsymbol{\lambda}, \tau)=\left(\Re a\left(\lambda_{1}, \tau\right), \Im a\left(\lambda_{1}, \tau\right), \ldots, \Re a\left(\lambda_{r}, \tau\right), \Im a\left(\lambda_{r}, \tau\right)\right)^{\prime} .
$$

Finally, the estimator of $a(\boldsymbol{\lambda}, \tau)$ and its bootstrap counterpart are denoted respectively by $\widetilde{a}(\boldsymbol{\lambda}, \tau)$ and $\widetilde{a}^{*}(\boldsymbol{\lambda}, \tau)$. The theorem below states the consistency of our bootstrap method for $a(\boldsymbol{\lambda}, \tau)$.

Theorem 3.2 Under the assumptions of Theorem 3.1

$$
\rho\left(\mathcal{L}\{\sqrt{n h}(\widetilde{a}(\boldsymbol{\lambda}, \tau)-a(\boldsymbol{\lambda}, \tau))\}, \mathcal{L}^{*}\left\{\sqrt{n h}\left(\widetilde{a}^{*}(\boldsymbol{\lambda}, \tau)-\mathrm{E}^{*} \widetilde{a}^{*}(\boldsymbol{\lambda}, \tau)\right)\right\}\right) \longrightarrow 0 \quad \text { in prob. },
$$

where $\rho$ is any metric metricizing convergence in distribution on $\mathbb{R}^{2 r}$. 


\subsection{Bootstrap confidence intervals}

Theorem 3.1 allows us to construct bootstrap percentile pointwise confidence intervals for the real and the imaginary part of $a(\lambda, \tau)$. Let us recall that in practice the asymptotic variance is almost impossible to estimate and hence standard asymptotic confidence intervals cannot be obtained. Bootstrap methods are an alternative approach which provides the confidence interval estimate for the unknown parameter. Below we discuss the construction of the $(1-\alpha) \%$ bootstrap percentile equal-tailed confidence interval for $\Re a(\lambda, \tau)$. The confidence interval for $\Im a(\lambda, \tau)$ can be obtained correspondingly.

Bootstrap pointwise confidence intervals

1. Let frequency $\lambda$ and shift $\tau$ be fixed. Repeat $B$ times the bootstrap algorithm described in Section 3 to obtain $B$ replicates of $\widetilde{a}^{*}(\lambda, \tau)$, i.e., $\widetilde{a}^{* 1}(\lambda, \tau), \ldots, \widetilde{a}^{* B}(\lambda, \tau)$.

2. Calculate the mean of the obtained values, i.e.,

$$
\bar{a}^{*}(\lambda, \tau)=\frac{1}{B} \sum_{i=1}^{B} \widetilde{a}^{* i}(\lambda, \tau) .
$$

3. For $i=1, \ldots, B$ calculate $y_{i}=\sqrt{n h}\left(\widetilde{a}^{* i}(\lambda, \tau)-\bar{a}^{*}(\lambda, \tau)\right)$ and sort the obtained values in ascending order to obtain $y_{(1)}, \ldots, y_{(B)}$.

4. Let $k_{1}=\lceil B \alpha / 2\rceil$ and $k_{2}=\lfloor B(1-\alpha / 2)\rfloor$. By $\lceil x\rceil$ and $\lfloor x\rfloor$ we denote the ceiling and the floor of the real number $x$. The $(1-\alpha) \%$ bootstrap percentile equal-tailed confidence interval for $\Re a(\lambda, \tau)$ is of the form

$$
\left(\Re \widetilde{a}(\lambda, \tau)-\frac{y_{\left(k_{2}\right)}}{\sqrt{n h}}, \Re \widetilde{a}(\lambda, \tau)-\frac{y_{\left(k_{1}\right)}}{\sqrt{n h}}\right) .
$$

In practice, for each $\tau$ many frequencies are considered. Thus, the simultaneous confidence intervals are of great importance. They are frequently met in real data applications (see, e.g., [12], [11]). To obtain them, the consistency of the bootstrap approach for smooth functions $H: \mathbb{R}^{2 r} \rightarrow \mathbb{R}^{s}, s \in \mathbb{N}$ of $a(\boldsymbol{\lambda}, \tau)$ needs to be shown, i.e.,

$$
\rho\left(\mathcal{L}\{\sqrt{n h}(H(\widetilde{a}(\boldsymbol{\lambda}, \tau))-H(a(\boldsymbol{\lambda}, \tau)))\}, \mathcal{L}^{*}\left\{\sqrt{n h}\left(H\left(\widetilde{a}^{*}(\boldsymbol{\lambda}, \tau)\right)-\mathrm{E}^{*} H\left(\widetilde{a}^{*}(\boldsymbol{\lambda}, \tau)\right)\right)\right\}\right) \longrightarrow 0 \quad \text { in prob. }
$$

This result is a direct consequence of Theorem 3.2 and the continuous mapping theorem (see [25]). Below we present the construction of the $(1-\alpha) \%$ bootstrap percentile equal-tailed simultaneous confidence intervals for $\Re a\left(\lambda_{1}, \tau\right), \ldots, \Re a\left(\lambda_{r}, \tau\right)$.

Bootstrap simultaneous confidence intervals

1. Let frequencies $\lambda_{1}, \ldots, \lambda_{r}$ and shift $\tau$ be fixed. Repeat $B$ times the bootstrap algorithm described in Section 3 to obtain $B$ replicates $\widetilde{a}^{* i}(\boldsymbol{\lambda}, \tau)=\left(\widetilde{a}^{* i}\left(\lambda_{1}, \tau\right), \ldots, \widetilde{a}^{* i}\left(\lambda_{r}, \tau\right)\right), i=1, \ldots, B$. 
2. For $j=1, \ldots, r$ calculate the means of the obtained values, i.e.,

$$
\bar{a}^{*}\left(\lambda_{j}, \tau\right)=\frac{1}{B} \sum_{i=1}^{B} \widetilde{a}^{* i}\left(\lambda_{j}, \tau\right) .
$$

3. For $i=1, \ldots, B$ calculate

$$
\begin{aligned}
& K_{\text {max }, i}=\sqrt{n h} \max _{j} \Re\left(\widetilde{a}^{* i}\left(\lambda_{j}, \tau\right)-\bar{a}^{*}\left(\lambda_{j}, \tau\right)\right), \\
& K_{\text {min }, i}=\sqrt{n h} \min _{j} \Re\left(\widetilde{a}^{* i}\left(\lambda_{j}, \tau\right)-\bar{a}^{*}\left(\lambda_{j}, \tau\right)\right) .
\end{aligned}
$$

4. Sort the obtained values in ascending order to obtain $K_{\max ,(1)}, \ldots, K_{\max ,(B)}$ and $K_{\min ,(1)}, \ldots, K_{\min ,(B)}$.

5. Let $k_{1}=\lceil B \alpha / 2\rceil$ and $k_{2}=\lfloor B(1-\alpha / 2)\rfloor$. The $(1-\alpha) \%$ bootstrap percentile equal-tailed simultaneous confidence intervals for $\Re a\left(\lambda_{1}, \tau\right), \ldots, \Re a\left(\lambda_{r}, \tau\right)$ are of the form

$$
\left(\Re \widetilde{a}_{n}\left(\lambda_{j}, \tau\right)-\frac{K_{\max ,\left(k_{2}\right)}}{\sqrt{n h}}, \Re \widetilde{a}_{n}\left(\lambda_{j}, \tau\right)-\frac{K_{\min ,\left(k_{1}\right)}}{\sqrt{n h}}\right)
$$

for $j=1, \ldots, r, \lambda_{1}, \ldots, \lambda_{r} \in \mathbb{R}$ and $\tau \in \mathbb{R}$. The confidence intervals for the imaginary case are defined correspondingly.

\section{Alternative bootstrap approach}

In this section we present a modification of the bootstrap approach presented in Section 3, which allows us to reduce bias of our bootstrap estimator for finite $n$. We use the idea introduced in [26]. The usual MBB is using $n-b+1$ blocks of the length $b$. The observations whose indices are between $b$ and $n-b$ are present in $b$ different blocks, while the observations from the beginning and the end of the sample appear less frequently. For example, the first observation is only in the block $B_{1}$. Politis and Romano showed that this leads to an increase in bias of the estimator. To reduce it, they proposed to treat the data as wrapped on the circle. Then each observation is present in the same number of blocks. Below we discuss how to apply this idea in our setting.

Let us recall that we apply bootstrap to the sample

$$
\left(\left(\ddot{b}_{1}(\tau), 1\right), \ldots,\left(\ddot{b}_{n}(\tau), n\right)\right) .
$$

By $B_{i}, i=1, \ldots, n$ we denote a block of observations that starts with observation $\left(\ddot{b}_{i}(\tau), i\right)$ and has length $b$. Note that for $i=1, \ldots, n-b+1$ we consider exactly the same blocks as defined in Section 3 . For $i=n-b+2, \ldots, n$ block $B_{i}$ is created by joining the observations from the end and the beginning of the sample to obtain a block of the required length. To be precise,

$B_{i}=\left(\left(\ddot{b}_{i}(\tau), i\right), \ldots,\left(\ddot{b}_{n}(\tau), n\right),\left(\ddot{b}_{1}(\tau), 1\right), \ldots,\left(\ddot{b}_{b-n+i-1}(\tau), b-n+i-1\right)\right) \quad$ for $\quad i=n-b+2, \ldots, n$.

Now each observation appears exactly in the same number of blocks. Below we present the circular version of the bootstrap algorithm presented in Section 3 .

Circular bootstrap approach 
1. Choose the block length $b<n$. Then our sample can be split into $l$ blocks of length $b$ and the remaining part of length $r<b$, i.e. $n=l b+r, r=0, \ldots, b-1$.

2. From the set $\left\{B_{1}, \ldots, B_{n}\right\}$ choose randomly with replacement $l+1$ blocks $B_{1}^{*}, \ldots, B_{l+1}^{*}$. The probability of choosing any block is equal to $1 / n$.

3. Join the selected $l+1$ blocks $\left(B_{1}^{*}, \ldots, B_{l+1}^{*}\right)$ and take the first $n$ observations to get the bootstrap sample $\left(\left(\ddot{b}_{1}^{*}(\tau), 1^{*}\right), \ldots,\left(\ddot{b}_{n}^{*}(\tau), n^{*}\right)\right)$ of the same length as the original one.

The bootstrap estimator $\widetilde{a}^{*}(\lambda, \tau)$ and its multidimensional version $\widetilde{a}^{*}(\boldsymbol{\lambda}, \tau)$ remain unchanged (see definitions in Section 3), except that they are constructed by random selections from the set of $n$ blocks.

As we mentioned above, Politis and Romano proposed a circular bootstrap idea to reduce the bias of their estimator. The authors considered stationary time series. Below we investigate if the proposed modification is bringing similar effects in our nonstationary case. Let $n=l b$, i.e., the sample size can be split into $l$ disjoint blocks of the length $b$. We have the following:

$$
\begin{aligned}
& \mathrm{E}^{*}\left(\widetilde{a}^{*}(\lambda, \tau)\right)=\mathrm{E}^{*}\left(\frac{1}{n} \sum_{k=1}^{n} \ddot{b}_{k}^{*}(\tau) \exp \left(-i \lambda k^{*} h\right)\right) \\
& =\frac{1}{n} \mathrm{E}^{*}\left(\sum_{k=1}^{b} \ddot{b}_{k}^{*}(\tau) \exp \left(-i \lambda k^{*} h\right)+\sum_{k=b+1}^{2 b} \ddot{b}_{k}^{*}(\tau) \exp \left(-i \lambda k^{*} h\right)+\cdots+\sum_{k=(l-1) b+1}^{l b} \ddot{b}_{k}^{*}(\tau) \exp \left(-i \lambda k^{*} h\right)\right) \\
& =\frac{1}{n} \sum_{j=1}^{l} \mathrm{E}^{*}\left(\sum_{k=(j-1) b+1}^{j b} \ddot{b}_{k}^{*}(\tau) \exp \left(-i \lambda k^{*} h\right)\right) \\
& =\frac{1}{n} \sum_{j=1}^{l} \frac{1}{n} \sum_{s=1}^{n}\left(\sum_{k=s}^{s+b-1} \ddot{b}_{k}(\tau) \exp (-i \lambda k h)\right)=\widetilde{a}(\lambda, \tau) .
\end{aligned}
$$

Note that the sum $\left(\sum_{k=s}^{s+b-1} \ddot{b}_{k}(\tau) \exp (-i \lambda k h)\right)$ is based on observations belonging to the block $B_{s}$. Each time when time index $k>n$ we take $k-n$ instead. Then we replace $\ddot{b}_{k}(\tau) \exp (-i \lambda k h)$ by $\ddot{b}_{k-n}(\tau) \exp (-i \lambda(k-n) h)$. For the sake of simplicity we decided not to introduce any additional notation to indicate this fact.

We showed that $\mathrm{E}^{*}\left(\widetilde{a}^{*}(\lambda, \tau)\right)=\widetilde{a}(\lambda, \tau)$, which means that $\widetilde{a}^{*}(\lambda, \tau)$ is the unbiased estimator of $\widetilde{a}(\lambda, \tau)$ conditionally on the observations. This property does not hold if the non-circular bootstrap algorithm is used. If the bootstrap sample $\left(\left(\ddot{b}_{1}^{*}(\tau), 1^{*}\right), \ldots,\left(\ddot{b}_{n}^{*}(\tau), n^{*}\right)\right)$ is obtained using the standard approach described in Section 3, we obtain the following:

$$
\begin{aligned}
& \mathrm{E}^{*}\left(\widetilde{a}^{*}(\lambda, \tau)\right)=\frac{1}{n} \sum_{j=1}^{l} \mathrm{E}^{*}\left(\sum_{k=(j-1) b+1}^{j b} \ddot{b}_{k}^{*}(\tau) \exp \left(-i \lambda k^{*} h\right)\right) \\
& =\frac{1}{n} \sum_{j=1}^{l} \frac{1}{n-b+1} \sum_{s=1}^{n-b+1}\left(\sum_{k=s}^{s+b-1} \ddot{b}_{k}(\tau) \exp (-i \lambda k h)\right) \\
& =\frac{1}{n-b+1}\left(n \widetilde{a}(\lambda, \tau)-\frac{1}{b} \sum_{j=1}^{b-1}(b-j)\left(\ddot{b}_{j}(\tau) \exp (-i \lambda j h)+\ddot{b}_{n-j+1}(\tau) \exp (-i \lambda(n-j+1) h)\right)\right)
\end{aligned}
$$


and hence $\widetilde{a}^{*}(\lambda, \tau)$ is biased.

Below we present consistency results for the circular bootstrap approach.

Theorem 4.1 Under assumptions of Theorem 3.1

$$
\rho\left(\mathcal{L}(\sqrt{n h}(\widetilde{a}(\lambda, \tau)-a(\lambda, \tau))), \mathcal{L}^{*}\left(\sqrt{n h}\left(\widetilde{a}^{*}(\lambda, \tau)-\widetilde{a}(\lambda, \tau)\right)\right)\right) \longrightarrow 0 \quad \text { in prob. }
$$

Theorem 4.2 Under the assumptions of Theorem 3.2

$$
\rho\left(\mathcal{L}\{\sqrt{n h}(\widetilde{a}(\boldsymbol{\lambda}, \tau)-a(\boldsymbol{\lambda}, \tau))\}, \mathcal{L}^{*}\left\{\sqrt{n h}\left(\widetilde{a}^{*}(\boldsymbol{\lambda}, \tau)-\widetilde{a}(\boldsymbol{\lambda}, \tau)\right)\right\}\right) \longrightarrow 0 \quad \text { in prob. }
$$

Finally, following the reasoning presented in Section 3.1 one may construct the pointwise and the simultaneous confidence intervals for Fourier coefficients using the circular version of our bootstrap approach.

\section{Simulation study}

In the following, we investigated how the procedures proposed by us work in practice. For that purpose we considered a signal

$$
X(t)=a(t) \cos (2 \pi t / 10)+\varsigma(t),
$$

where $a(t)$ is a stationary zero-mean Gaussian process with standard deviation equal to 0.1 and $\varsigma(t)$ is a $\mathrm{AR}(2)$ correlated additive Gaussian noise of the form

$$
\varsigma(t)=0.5 \varsigma(t-1)+0.25 \varsigma(t-2)+\epsilon(t),
$$

where $\epsilon(t)$ are iid random variables from the normal distribution with mean equal to 0 and standard deviation equal to 0.05 .

The set of the second order cyclic frequencies is of the form

$$
\Lambda=\{-0.2 H z, 0 H z, 0.2 H z\} .
$$

In each period of length $10 \mathrm{~s}$ we collected 100 observations. We assumed that we observed 100 of periods and hence the length of the sample was $n=10000$ observations. To detect significant frequencies, we constructed $95 \%$ bootstrap pointwise and simultaneous confidence intervals (see Section 3.1) for $\operatorname{Re}(a(\lambda, 0))$ and $\operatorname{Im}(a(\lambda, 0))$. Due to the symmetry of the cyclic spectrum, we restricted our study to $\lambda \in\{0 \mathrm{~Hz}, 0.01 \mathrm{~Hz}, \ldots, 0.5 \mathrm{~Hz}\}$, using the circular version of our bootstrap approach (see Section 4 ). We took two block lengths $b \in\{20,100\}$, which were equal approximately to $\sqrt[3]{n}$ and $\sqrt{n}$. The number of bootstrap samples $B=500$. Moreover, we considered two types of random perturbations. In the first case $U_{i}$ were iid random variables from the uniform distribution on $(-h / 2, h / 2)$, while in the second one $U_{i}$ were iid from the truncated normal distribution (mean and standard deviation respectively equal to 0 and 0.01) i.e., distribution restricted to the interval $(-h / 2, h / 2)$.

The obtained results are presented in Figures 1-2 (uniform distribution case) and 3-4 (truncated normal distribution case). Independently of the considered distribution and the chosen block length using simultaneous confidence intervals, we always detected two frequencies, i.e., $0 \mathrm{~Hz}$ and $0.2 \mathrm{~Hz}$, which were the true frequencies. The frequency $\lambda$ was detected as significant when at least one of the corresponding confidence intervals for $\operatorname{Re}(a(\lambda, 0))$ and $\operatorname{Im}(a(\lambda, 0))$ did not contain 0 value, which denoted that $a(\lambda, 0) \neq 0$. The pointwise confidence intervals provided the same results, except for one case. For $b=100$ with the truncated normal distribution, we detected an additional frequency of $0.11 \mathrm{~Hz}$. The simultaneous confidence intervals proposed by us are less sensitive to the block length choice and seem to be a convenient 


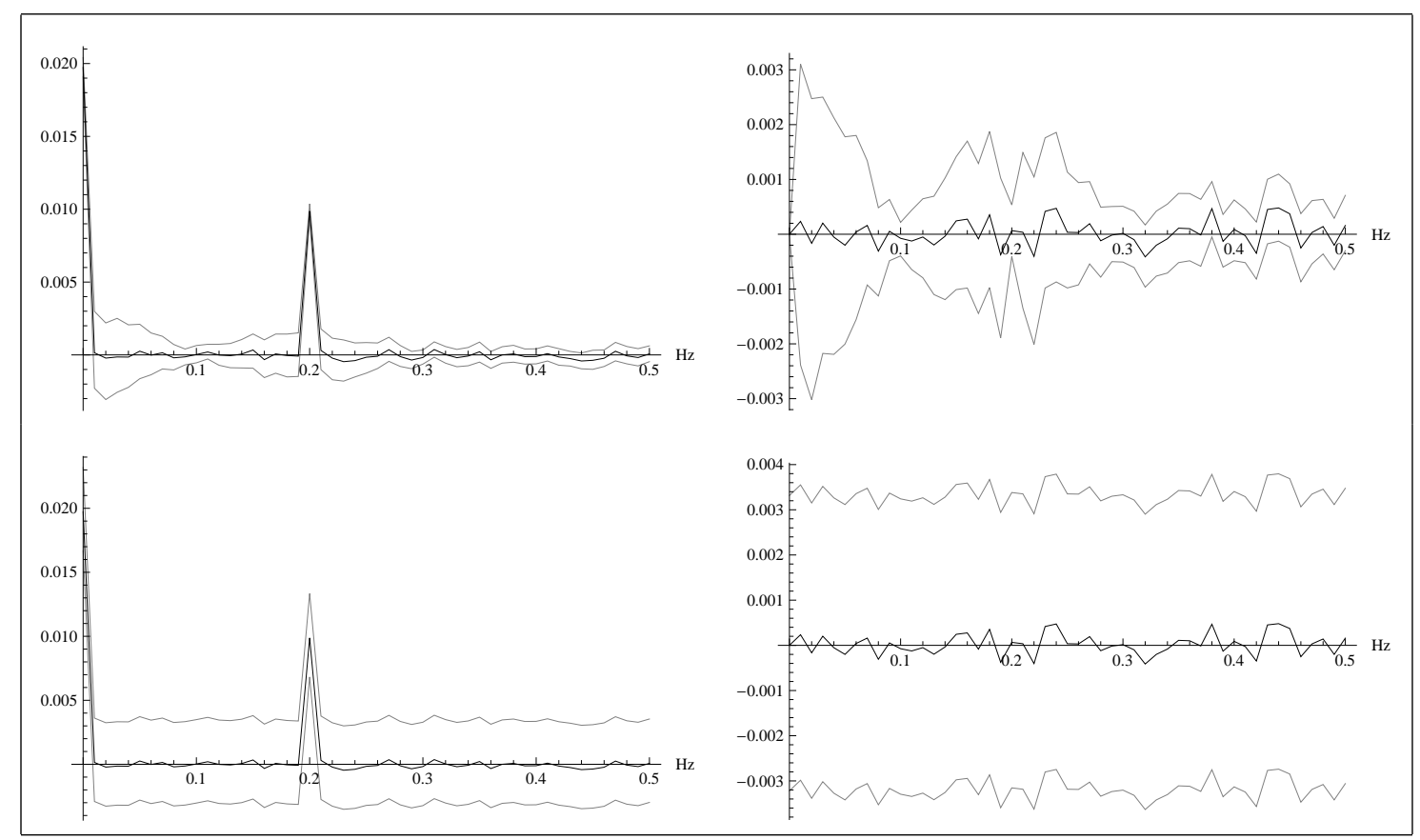

Figure 1: Results for uniform distribution. Grey color: pointwise (first row) and simultaneous (second row) confidence intervals for $\operatorname{Re}(a(\lambda, 0))$ (left column) and $\operatorname{Im}(a(\lambda, 0))$ (right column), $\lambda \in\{0 H z, 0.01 H z, \ldots, 0.5 H z\}$ and $b=100$. Black color: estimators of $\operatorname{Re}(a(\lambda, 0))$ and $\operatorname{Im}(a(\lambda, 0))$. Nominal coverage probability is $95 \%$.

tool for frequency detection.

Finally, to check effectiveness of our approach we compared performance of our bootstrap technique with the Monte Carlo (MC) evaluation. The values of the all parameters were the same. The number of the MC replicates was 500. The results obtained with the MC method are presented in Figures 5-6. Using the simultaneous confidence intervals independently of the chosen distribution, two frequencies were detected: $0 \mathrm{~Hz}$ and $0.2 \mathrm{~Hz}$. Thus, the MC approach and our bootstrap method provided exactly the same results, detecting precisely the true frequencies. However, in the case of the pointwise confidence intervals our approach was superior to the MC one. With the MC method, we detected the following set of frequencies (in $\mathrm{Hz}$ ):

$$
\begin{array}{r}
\{0,0.01,0.07,0.09,0.1,0.13,0.14,0.18,0.19,0.2,0.23,0.25,0.26,0.27,0.29,0.31 \\
0.32,0.34,0.37,0.38,0.39,0.41,0.43,0.45,0.47\}
\end{array}
$$

in a case of the truncated normal distribution and

$$
\begin{array}{r}
\{0,0.01,0.02,0.05,0.07,0.09,0.1,0.11,0.13,0.17,0.18,0.2,0.21,0.23,0.28,0.3,0.31 \\
0.35,0.4,0.41,0.43,0.45,0.46,0.49,0.5\}
\end{array}
$$

in the case of the uniform distribution. We obtained many frequencies that were not the true frequencies. Thus, our bootstrap approach seems to be more suitable for practical applications. 


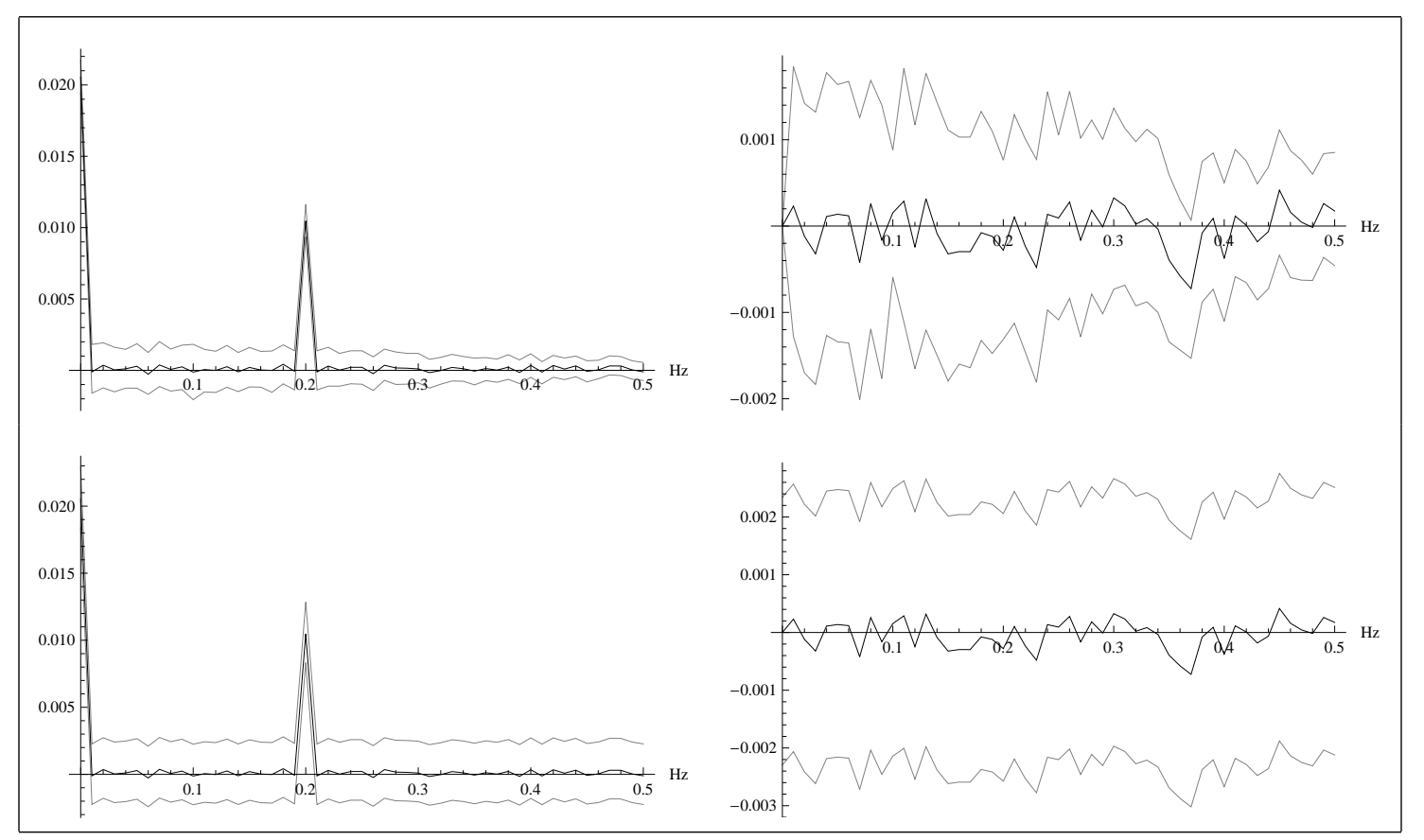

Figure 2: Results for uniform distribution. Grey color: pointwise (first row) and simultaneous (second row) confidence intervals for $\operatorname{Re}(a(\lambda, 0))$ (left column) and $\operatorname{Im}(a(\lambda, 0))$ (right column), $\lambda \in\{0 H z, 0.01 H z, \ldots, 0.5 H z\}$ and $b=20$. Black color: estimators of $\operatorname{Re}(a(\lambda, 0))$ and $\operatorname{Im}(a(\lambda, 0))$. Nominal coverage probability is $95 \%$.

\section{Analysis of Fourier spectrum of gear vibration signal}

\subsection{Fault detection of a gear system by spectral analysis}

Spectral analysis is a natural tool for the processing of signals in mechanics. In general, the vibration signal taken from a rotating machine is a composition that responds to many different excitation forces. The aim of spectral analysis is to be able to dissociate and identify vibratory sources according to the kinematic characteristics of the various constituent elements and their speed of rotation (or their frequency of movement).

The vibrations resulting from a single stage reduction (Figure 7) are mainly due to the deviation of the position of the wheels with respect to a perfect rotational movement. A number of meshing signal models were presented in the literature in [27], [28] and [29]. These models more or less translate the reality of the measured signals. The signal may be summarized as follows: the vibration, called the meshing signal, is periodic and its frequency (meshing frequency) is equal to the frequency of rotation of one of the two wheels multiplied by the number of teeth of this wheel. Moreover, this meshing signal is modulated in amplitude and frequency by both a periodic signal of period equal to the period of rotation of the pinion and a periodic signal of period equal to the period of rotation of the wheel. In general the frequency modulation is much less important than the amplitude modulation.

By neglecting the frequency modulations the model established by Capdessus and Sidahmed in [30] can be used:

$$
s_{c}(t)=\sum_{n=-\infty}^{\infty} s_{e}\left(t-n \tau_{e}\right)\left(1+\sum_{m=-\infty}^{\infty} s_{p_{1}}\left(t-m \tau_{p_{1}}\right)+\sum_{p=-\infty}^{\infty} s_{p_{2}}\left(t-p \tau_{p_{2}}\right)\right),
$$




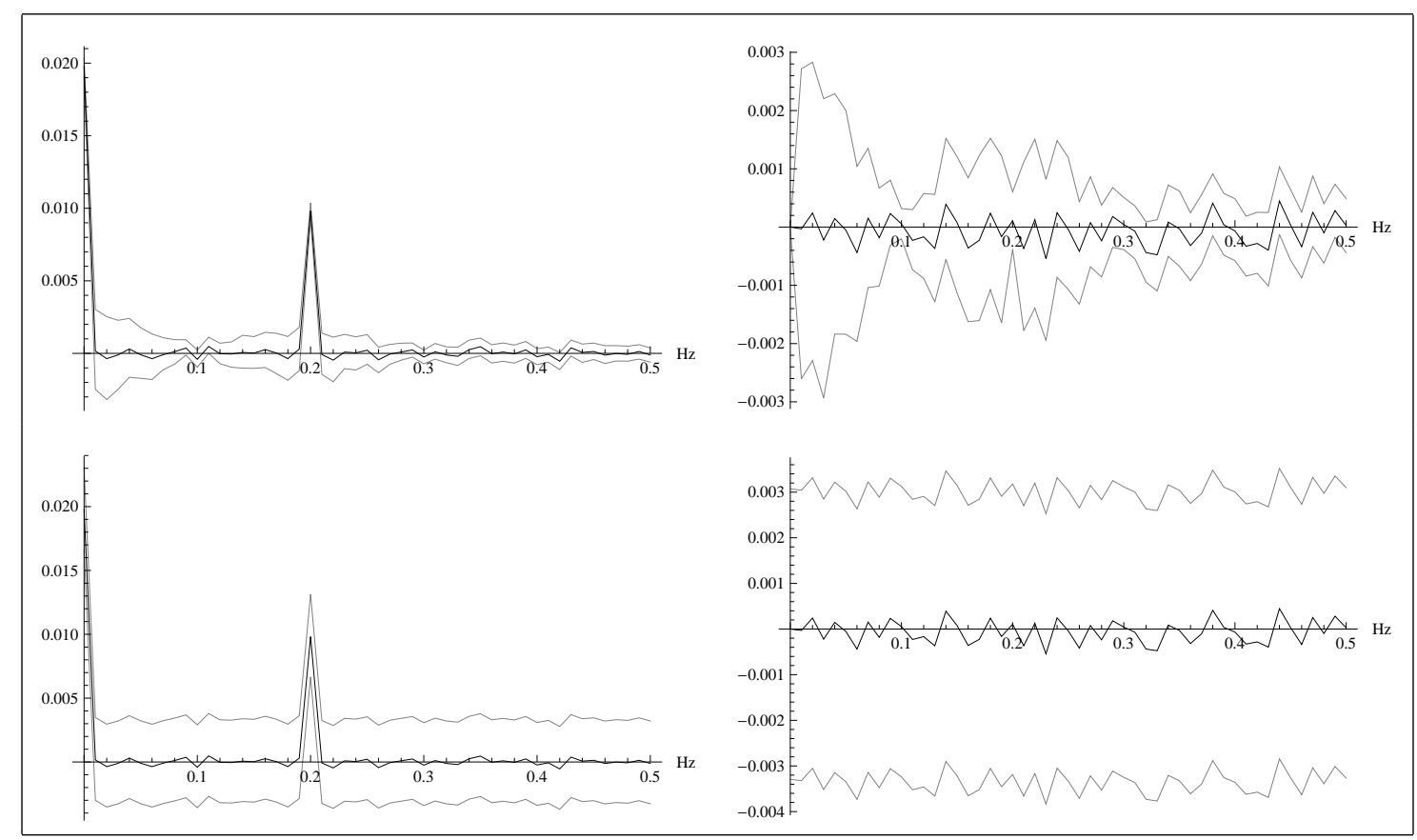

Figure 3: Results for truncated normal distribution. Grey color: pointwise (first row) and simultaneous (second row) confidence intervals for $\operatorname{Re}(a(\lambda, 0))$ (left column) and $\operatorname{Im}(a(\lambda, 0))$ (right column), $\lambda \in\{0 \mathrm{~Hz}, 0.01 \mathrm{~Hz}, \ldots, 0.5 \mathrm{~Hz}\}$ and $b=$ 100. Black color: estimators of $\operatorname{Re}(a(\lambda, 0))$ and $\operatorname{Im}(a(\lambda, 0))$. Nominal coverage probability is $95 \%$.

where $\tau_{e}$ is the meshing period, $\tau_{p_{1}}$ is the rotation period of wheel $1, \tau_{p_{2}}$ is the rotation period of wheel $2, s_{e}(t)$ is the vibratory meshing signal, $s_{p_{1}}(t)$ is the vibratory signal produced by wheel 1 and $s_{p_{2}}(t)$ is the vibratory signal produced by wheel 2 .

The most striking characteristic of the signal is the amplitude modulation due to the rotation of the wheels. Here we have a bidimensional spectrum which is composed by a family of lines of frequency $k f_{e}=k / \tau_{e}$ due to the fundamental frequency and harmonics of the meshing signal (Figure 8). This family of lines is spread over a large part of the spectrum, because the nature of the meshing signal is of a broadband type. Moreover, the amplitude modulation results in the presence of lateral bands around the meshing harmonics at multiple distances of $f_{1}=1 / \tau_{p_{1}}$ for the modulation due to wheel 1 and $f_{2}=1 / \tau_{p_{2}}$ for the modulation due to wheel 2 .

The detection and localization of the teeth defects of the wheels go through the statistical estimation of the characteristic frequencies. In the next section we estimated the frequency of meshing a vibratory signal from a gear system.

\subsection{Real vibratory signal}

In this section we applied our bootstrap technique to a vibratory signal. The recordings were carried out at CETIM on a gear system with a train of gearing with a ratio of $20 / 21$ functioning continuously until its destruction. The gearbox was running at $120 \%$ of the nominal power. The test was of length 12 days with a daily mechanical appraisal; measurements were collected every $24 \mathrm{~h}$. These signals were used on several occasions to demonstrate diagnostic procedures [30] and [31]. In the considered experiment the accelerometer signal was collected in ideally equally spaced time instances, which were randomly disturbed. This corresponds to the jitter sampling scheme introduced in Section 2. The non-disturbed 


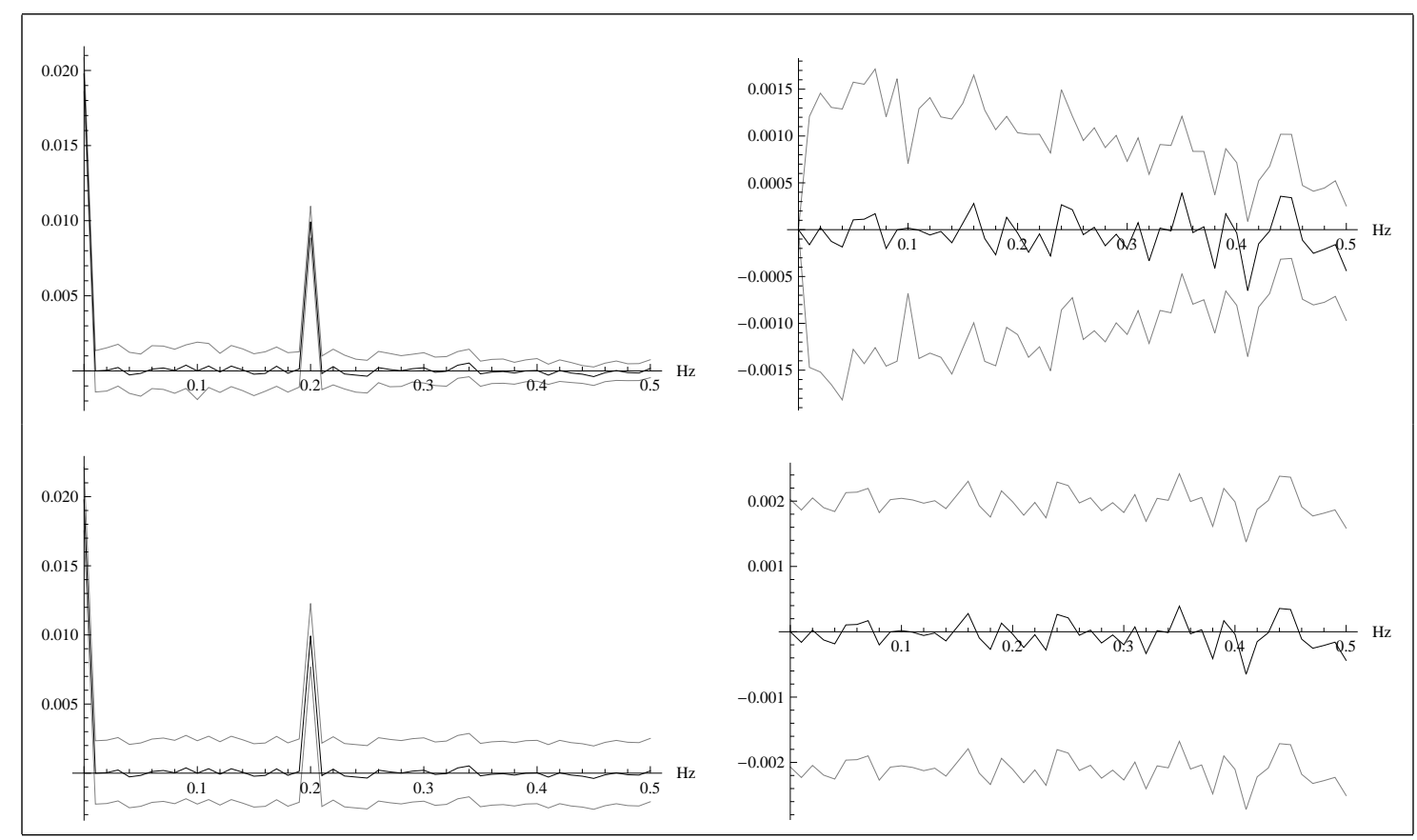

Figure 4: Results for truncated normal distribution. Grey color: pointwise (first row) and simultaneous (second row) confidence intervals for $\operatorname{Re}(a(\lambda, 0))$ (left column) and $\operatorname{Im}(a(\lambda, 0))$ (right column), $\lambda \in\{0 H z, 0.01 H z, \ldots, 0.5 H z\}$ and $b=20$. Black color: estimators of $\operatorname{Re}(a(\lambda, 0))$ and $\operatorname{Im}(a(\lambda, 0))$. Nominal coverage probability is $95 \%$.

time step $h$ was set $1 / 5000$ sec. The disturbances were generated from uniform distribution with mean 0 and standard deviation $h / 2$. The number of observations of the signal was 30000 . In this signal, we had characteristic frequencies at $340 \mathrm{~Hz}$ and its harmonics. Our aim was to detect the meshing frequency of $340 \mathrm{~Hz}$. Tracking the amplitude of this frequency can be used to detect an anomaly. In the following, we will not deal with the case of fault detection which has already been published in [32]. The signal is presented in Figure 9. Note that the observed values of the signal range from -10000 to 8000 . Thus, to simplify the calculation and the presentation of the results we divided them by 1000 .

To detect significant frequencies, we constructed $95 \%$ bootstrap pointwise and simultaneous confidence intervals (see Section 3.1) for $\operatorname{Re}(a(\lambda, 0))$ and $\operatorname{Im}(a(\lambda, 0)), \lambda \in\{300 H z, 310 H z, \ldots, 700 H z\}$, using the circular version of our bootstrap approach (see Section 4). As in Section 5, we used two block lengths $b \in\{30,200\}$, which were approximately equal to $\sqrt[3]{n}$ and $\sqrt{n}$. The number of bootstrap samples $B=500$. The results are presented in Figures 10-11.

Independently of the chosen block length $b$ and used type of confidence intervals, we always successfully detected a frequency $340 \mathrm{~Hz}$ and its harmonic $680 \mathrm{~Hz}$, which means that our bootstrap approach allowed to point out precisely the true frequencies.

\section{Summary and conclusions}

In this paper we considered the continuous CS signal which is observed in instants that are randomly disturbed. We focused on the second-order analysis, i.e., we dealt with the autocovariance function of the signal. We provided two bootstrap approaches based on the MBB method that enabled us to construct the pointwise and the simultaneous confidence intervals for the Fourier coefficients of the autocovariance function. We used these confidence bands to detect the second-order significant frequencies i.e., such 


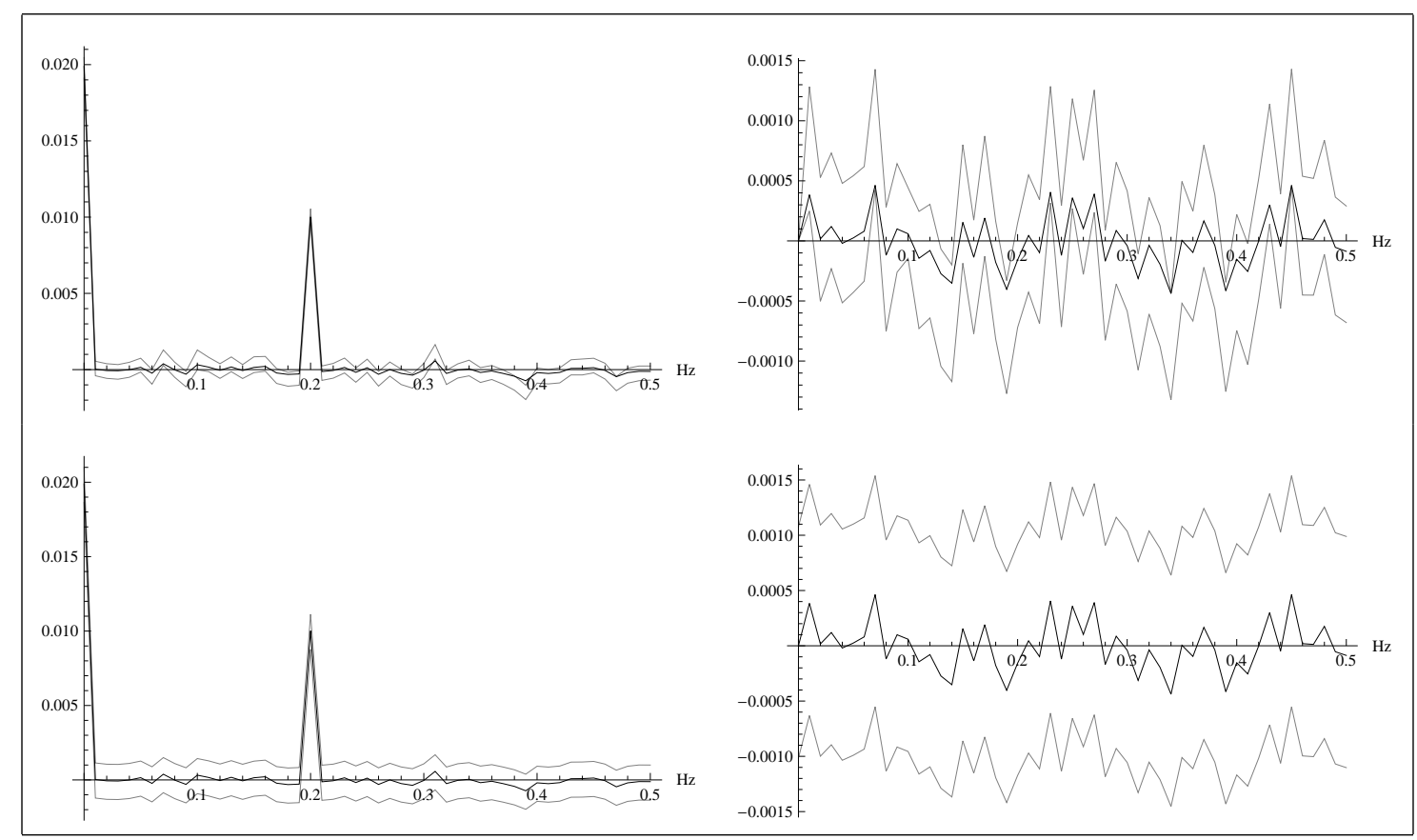

Figure 5: Monte Carlo method results for truncated normal distribution. Grey color: pointwise (first row) and simultaneous (second row) confidence intervals for $\operatorname{Re}(a(\lambda, 0))$ (left column) and $\operatorname{Im}(a(\lambda, 0))$ (right column), $\lambda \in$ $\{0 H z, 0.01 H z, \ldots, 0.5 H z\}$. Black color: estimators of $\operatorname{Re}(a(\lambda, 0))$ and $\operatorname{Im}(a(\lambda, 0))$. Nominal coverage probability is $95 \%$.

frequencies for which the Fourier coefficients are nonzero.

The efficiency of the methods developed in the paper was confirmed by the results obtained from our simulated and real data examples. The performed simulation study showed that our approach worked well, independently of the distribution of instants of random perturbation. The application of the bootstrap technique to the real vibratory gearbox signals allowed to evaluate approximate confidence intervals that can be used for frequency detection when the sampling is perturbed.

In real data applications it happens that jitter is causing a change of order among observations. In our approach we assumed that jitter is present, but order of observations is kept, e.g., random perturbations are quite small. Thus, in the future it would be interesting to extend our approach to more general cases, i.e., to weaken our assumption (ii).

Finally, in the future we would like to continue our work to apply it to compressive sensing, where one observes a random sampling and has only a few samples. Using some tools designed for CS processes together with bootstrap approach, we would like to provide some new solutions for compressive sensing.

\section{Appendix}

For the sake of simplicity and clarity we assume that the sample size $n$ is an integer multiple of the block length $b(n=l b)$. Moreover, since the proofs for standard and circular bootstrap approaches follow exactly the same steps, we present details only for the circular method.

\section{Proof of Theorems 4.1 and 4.2}

\section{Proof:}




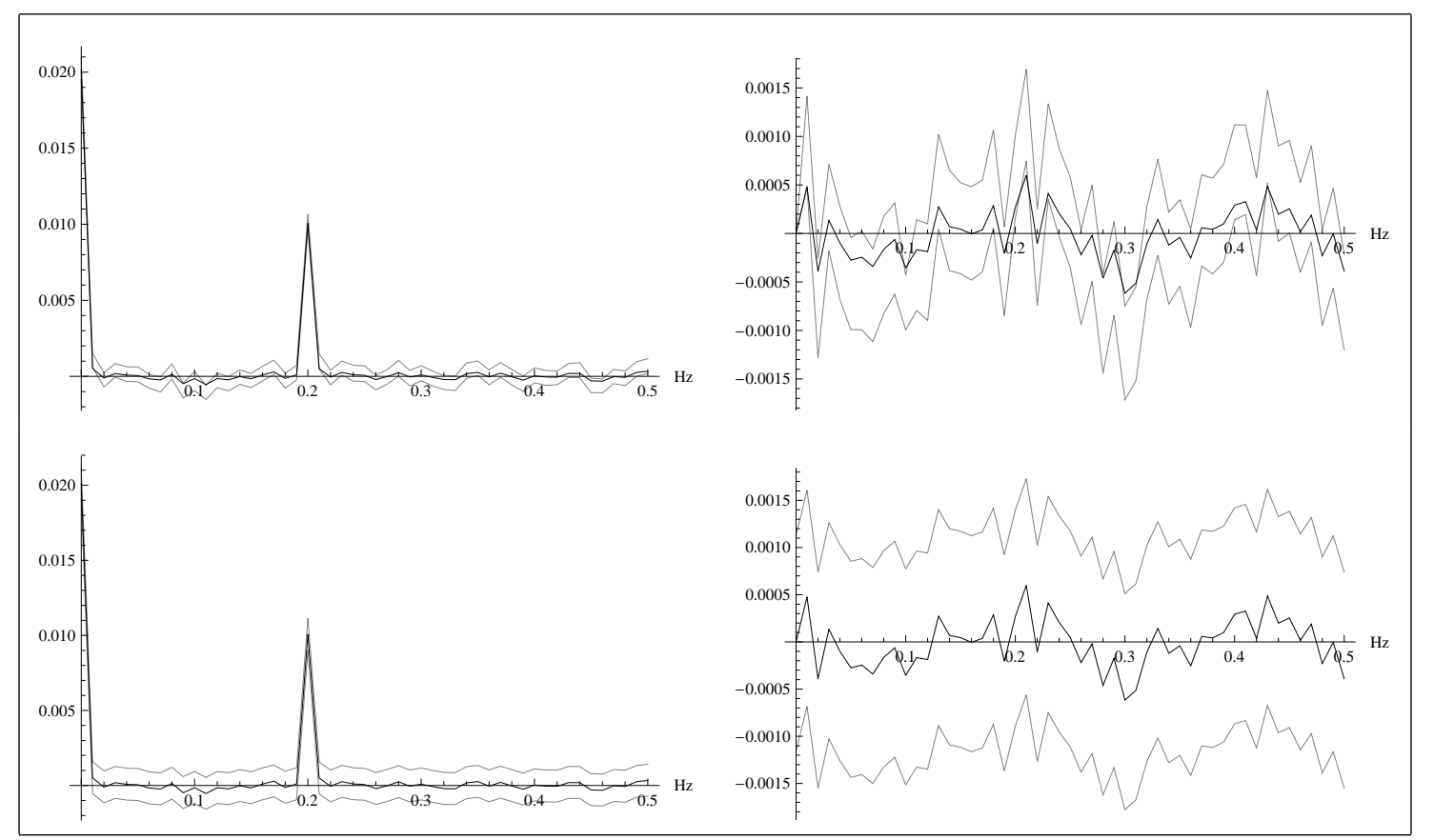

Figure 6: Monte Carlo method results for uniform distribution. Grey color: pointwise (first row) and simultaneous (second row) confidence intervals for $\operatorname{Re}(a(\lambda, 0))$ (left column) and $\operatorname{Im}(a(\lambda, 0))$ (right column), $\lambda \in\{0 H z, 0.01 H z, \ldots, 0.5 H z\}$. Black color: estimators of $\operatorname{Re}(a(\lambda, 0))$ and $\operatorname{Im}(a(\lambda, 0))$. Nominal coverage probability is $95 \%$.

We need to show that the circular bootstrap method is consistent, i.e., that

$$
\rho\left(\mathcal{L}(\sqrt{n h}(\widetilde{a}(\lambda, \tau)-a(\lambda, \tau))), \mathcal{L}^{*}\left(\sqrt{n h}\left(\widetilde{a}^{*}(\lambda, \tau)-\widetilde{a}(\lambda, \tau)\right)\right)\right) \longrightarrow 0 \quad \text { in prob. }
$$

as $n \rightarrow \infty$. At first we prove the theorem for $\Re\left(\widetilde{a}^{*}(\lambda, \tau)\right)$. Since $\widetilde{a}^{*}(\lambda, \tau)$ is unbiased (see Section 4$)$, we have that

$$
\mathrm{E}^{*}\left(\Re \widetilde{a}^{*}(\lambda, \tau)\right)=\Re \widetilde{a}(\lambda, \tau) .
$$

By $\widetilde{Z}_{t, b}$ we denote

$$
\widetilde{Z}_{t, b}=\sum_{s=1}^{b} \ddot{b}_{(j-1) b+s}(\tau) \cos (\lambda((j-1) b+s) h) .
$$

Using new notation we can rewrite the estimator $\widetilde{a}(\lambda, \tau)$ as follows:

$$
\widetilde{a}(\lambda, \tau)=\frac{1}{n} \sum_{j=1}^{l} \widetilde{Z}_{j, b} .
$$

Correspondingly,

$$
\widetilde{a}^{*}(\lambda, \tau)=\frac{1}{n} \sum_{j=1}^{l} \widetilde{Z}_{j, b}^{*}
$$




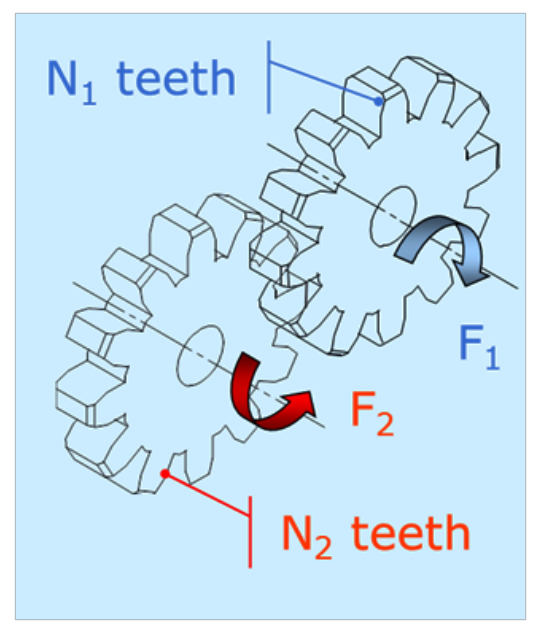

Figure 7: Single stage gear system. Green and red arrows provide information about direction of rotation of wheels. $F_{1}=f_{p_{1}}$ and $F_{2}=f_{p_{2}}$ are rotation frequencies of wheel 1 and 2 , respectively.

where

$$
\widetilde{Z}_{t, b}^{*}=\sum_{s=1}^{b} \ddot{b}_{(j-1) b+s}^{*}(\tau) \cos \left(\lambda((j-1) b+s)^{*} h\right),
$$

Additionally, let

$$
Z_{t, b}=\widetilde{Z}_{t, b}-\mathrm{E}\left(\widetilde{Z}_{t, b}\right)
$$

and its bootstrap version

$$
Z_{t, b}^{*}=\widetilde{Z}_{t, b}^{*}-\mathrm{E}^{*}\left(\widetilde{Z}_{t, b}^{*}\right)
$$

To show consistency, we use Corollary 2.4.8 in Araujo and Giné(1980). We need to show that for any $\nu>0$

$$
\begin{aligned}
& \sum_{k=0}^{l-1} P^{*}\left(\frac{1}{\sqrt{n h}}\left|Z_{1+k b, b}^{*}\right|>\nu\right) \longrightarrow 0 \text { in prob., } \\
& \sum_{k=0}^{l-1} \mathrm{E}^{*}\left(\frac{1}{\sqrt{n h}} Z_{1+k b, b}^{*} \mathbf{1}_{\left\{\left|Z_{1+k b, b}^{*}\right|>\sqrt{n h} \nu\right\}}\right) \longrightarrow 0 \text { in prob., } \\
& \sum_{k=0}^{l-1} \operatorname{Var}^{*}\left(\frac{1}{\sqrt{n h}} Z_{1+k b, b}^{*} \mathbf{1}_{\left.\left\{\left|Z_{1+k b, b}^{*}\right| \leq \sqrt{n h} \nu\right\}\right) \longrightarrow \sigma_{1}^{2} \text { in prob. }}\right.
\end{aligned}
$$

Since the main steps of the proof are the same as the proof of Theorem 2 in [11], we skip technical details. The same reasoning can be applied for the imaginary case. Finally, the consistency of $\widetilde{a}^{*}(\lambda, \tau)$, which is the two-dimensional vector, is a consequence of the Cramér-Wold device. The same argument is used to prove Theorem 4.2 . 


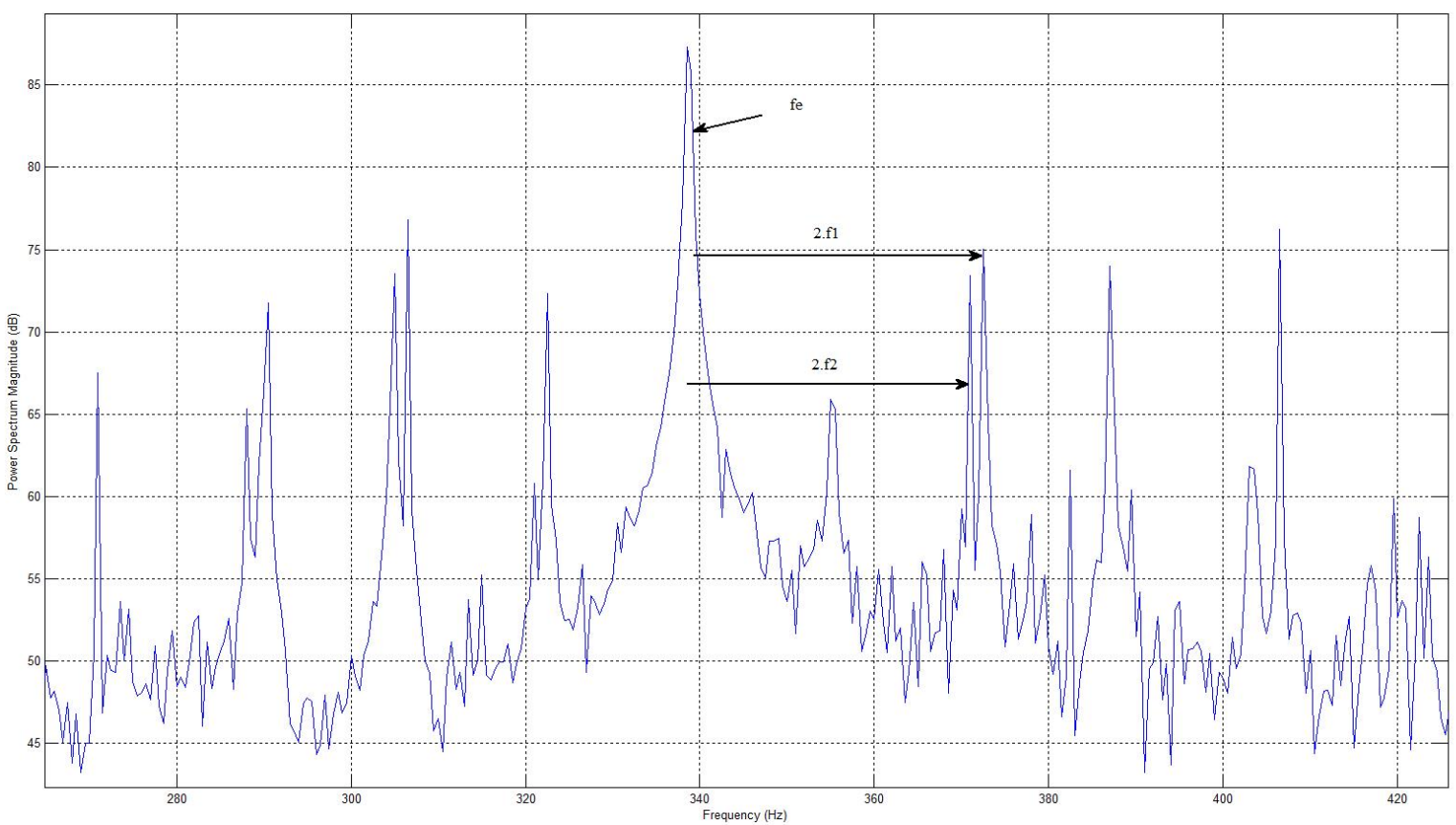

Figure 8: Fourier spectrum of gear vibration signal.

\section{References}

[1] H.L. Hurd, A.G. Miamee, Periodically Correlated Random Sequences: Spectral,Theory and Practice, Wiley, 2007.

[2] A.S. Besicovitch, Almost Periodic Functions, University Press, Cambridge, 1932.

[3] A. Napolitano, Generalizations of Cyclostationary Signal Processing: Spectral Analysis and Applications, Wiley-IEEE Press, 2012.

[4] H. Hurd, J. Leśkow, Strongly consistent and asymptotically normal estimation of the covariance for almost periodically correlated processes, Statist. Decisions 10 (1992) 201-225.

[5] B. Efron, Bootstrap methods: another look at the jacknife, Ann Statist. 7 (1979) 1-26.

[6] H. Künsch, The jackknife and the bootstrap for general stationary observations, Ann. Statist. 17 (1989) 1217-1241.

[7] R. Liu, K. Singh, Moving block jackknife and bootstrap capture weak dependence. Exploring the Limits of Bootstrap, Wiley Ser. Probab. Math. Statist. Probab. Math. Statist., Wiley, New York, pp 225-248, 1992.

[8] A.E. Dudek, J. Leśkow, E. Paparoditis, D. Politis, A generalized block bootstrap for seasonal time series, J. Time Ser. Anal. 35 (2014) 89-114. 


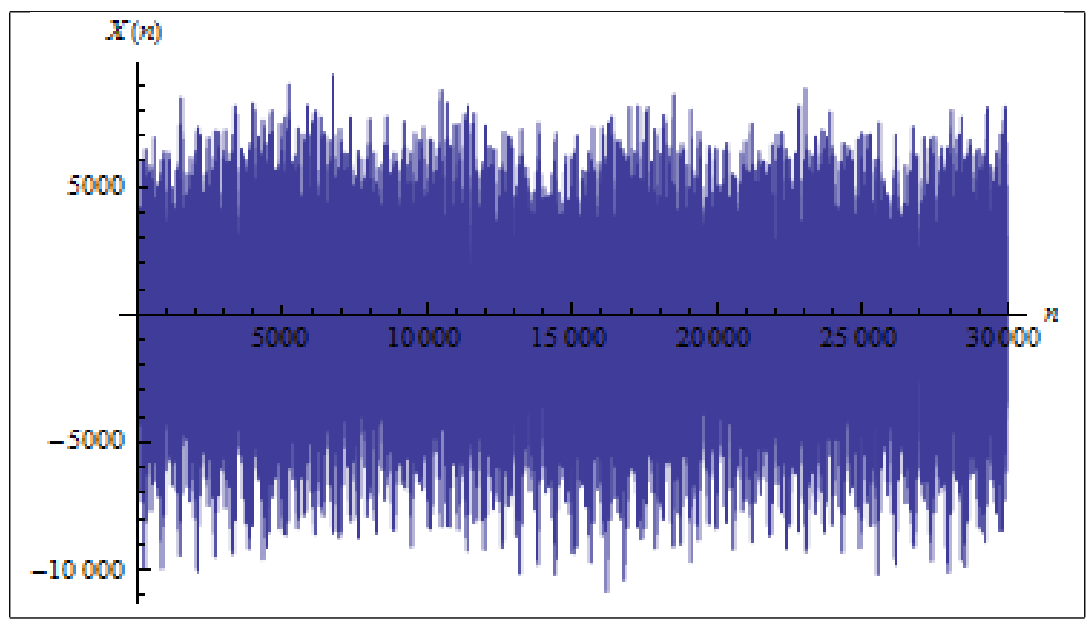

Figure 9: Vibratory signal of gear system.

[9] A.E. Dudek, E. Paparoditis, D. Politis, Generalized seasonal tapered block bootstrap, Statistics and Probability Letters 115 (2016) 27-35.

[10] R. Synowiecki, Consistency and application of moving block bootstrap for nonstationary time series with periodic and almost periodic structure, Bernoulli 13(4) (2007) 1151-1178.

[11] A.E. Dudek, Circular block bootstrap for coefficients of autocovariance function of almost periodically correlated time series, Metrika 78(3) (2015) 313-335.

[12] A.E. Dudek, S. Maiz, M. Elbadaoui, Generalized seasonal block bootstrap in frequency analysis of cyclostationary signals, Signal Process. 104C (2014) 358-368.

[13] D. Dehay, A.E. Dudek, Bootstrap method for Poisson sampling almost periodic process, J. Time Ser. Anal. 36(3) (2015) 327-351.

[14] D. Dehay, A.E. Dudek, Bootstrap for the second-order analysis of Poisson-sampled almost periodic processes, Electronic Journal of Statistics, 11(1) (2017) 99-147.

[15] M. Verbeke, P. Rombouts, A. Vyncke, G. Torfs, Influence of Jitter on Limit Cycles in Bang-Bang Clock and Data Recovery Circuits, IEEE Transactions on Circuits and Systems I 62 (2015) 14631471.

[16] D. Vercaemer, P. Rombouts, Analyzing the Effect of Clock Jitter on Self-Oscillating Sigma Delta Modulators, IEEE Transactions on Circuits and Systems I 63 (2016) 200-210.

[17] Y. Shim, D. Oh, System level modeling of supply noise induced jitter for high speed clock forwarding interfaces, IEEE Electromagnetic Compatibility Magazine 5 (2016) 117-122.

[18] M. El Badaoui, F. Bonnardot, Impact of the non-uniform angular sampling on mechanical signals, Mechanical Systems and Signal Processing 44 (2014) 199-210.

[19] F. Bonnardot, M. El Badaoui, R.B. Randall, J. Daniere, F. Guillet, Use of the acceleration signal of a gearbox in order to perform angular resampling (with limited speed fluctuation), Mechanical Systems and Signal Processing 19 (2005) 766-785. 


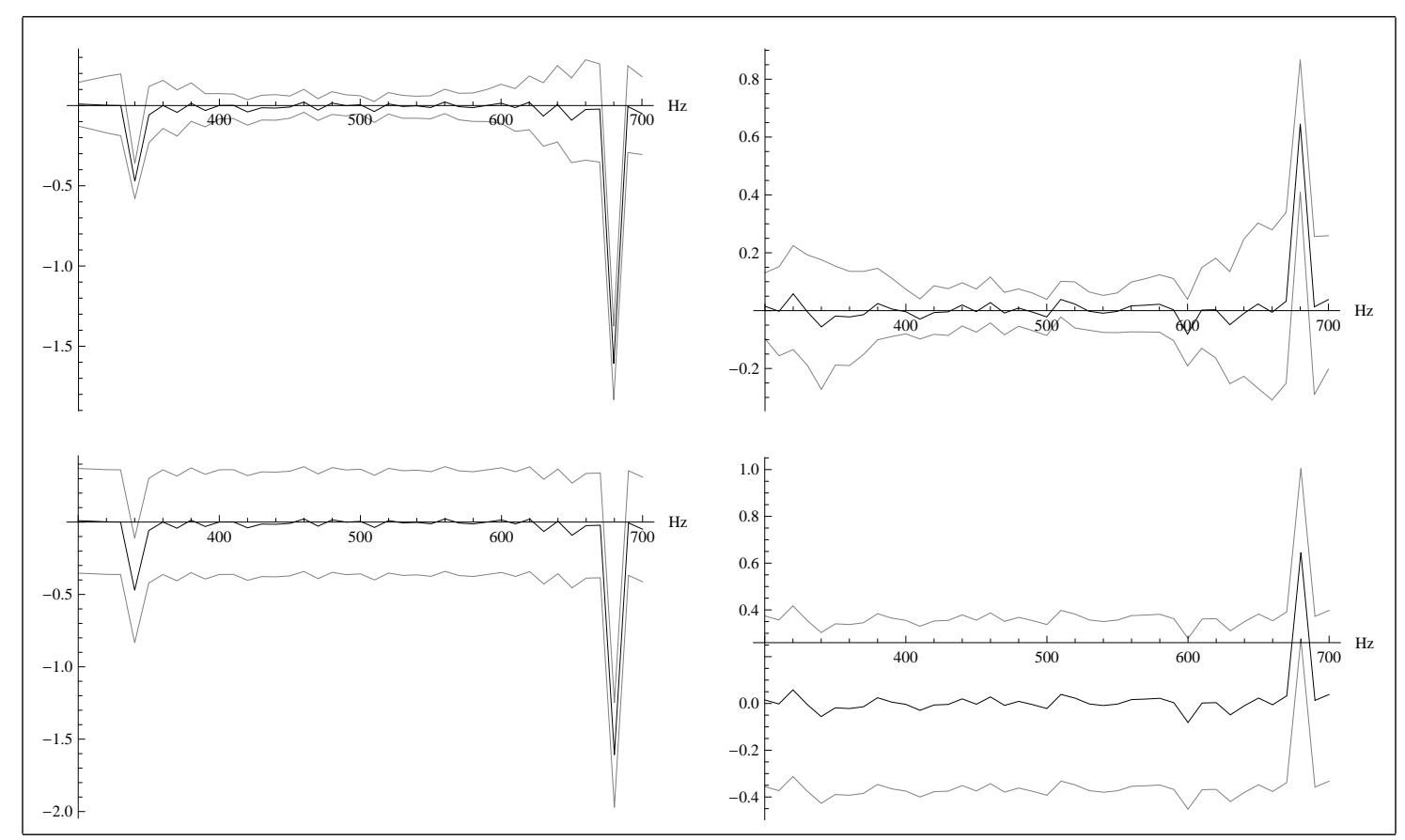

Figure 10: Grey color: pointwise (first row) and simultaneous (second row) confidence intervals for $\operatorname{Re}(a(\lambda, 0))($ left column) and $\operatorname{Im}(a(\lambda, 0))$ (right column), $\lambda \in\{300 \mathrm{~Hz}, 310 \mathrm{~Hz}, \ldots, 700 \mathrm{~Hz}\}$ and $b=200$. Black color: estimators of $\operatorname{Re}(a(\lambda, 0))$ and $\operatorname{Im}(a(\lambda, 0))$. Nominal coverage probability is $95 \%$.

[20] Q. Leclere, L.Pruvost, E. Parizet, Angular and temporal determinism of rotating machine signals: The diesel engine case, Mechanical Systems and Signal Processing 24 (2010) 2012-2020.

[21] D. Dehay, V. Monsan, Discrete periodic sampling with jitter and almost periodically correlated processes, Statistical Inference for Stochastic Processes 10 (2007) 223-253.

[22] D. Dehay, Asymptotic behavior of estimators of cyclic functional parameters for some nonstationary processes, Statist. Decisions 13 (1995) 273-286.

[23] P. Doukhan, Mixing : Properties and Examples, Lecture Notes in Statistics 85, Springer, New York, 1994.

[24] R. Synowiecki, Metody resamplingowe w dziedzinie czasu dla niestacjonarnych szeregów czasowych o strukturze okresowej i prawie okresowej, Dissertation, AGH University of Science and Techniology, Krakow, Poland, http://winntbg.bg.agh.edu.pl/rozprawy2/10012/full10012.pdf, 2008.

[25] P. Billingsley, Convergence of Probability Measures, Wiley Series in Probability and Mathematical Statistics, John Wiley \& Sons. Inc., New York, 1999.

[26] D.N. Politis, J.P. Romano, A circular block-resampling procedure for stationary data, Exploring the Limits of Bootstrap, Wiley Ser. Probab. Math. Statist. Probab. Math. Statist. Wiley, New York, pp 263-270, 1992.

[27] R.B. Randall, A new method of modelling gear faults, Journal of Mechanical Design 104 (1982) 259-267. 


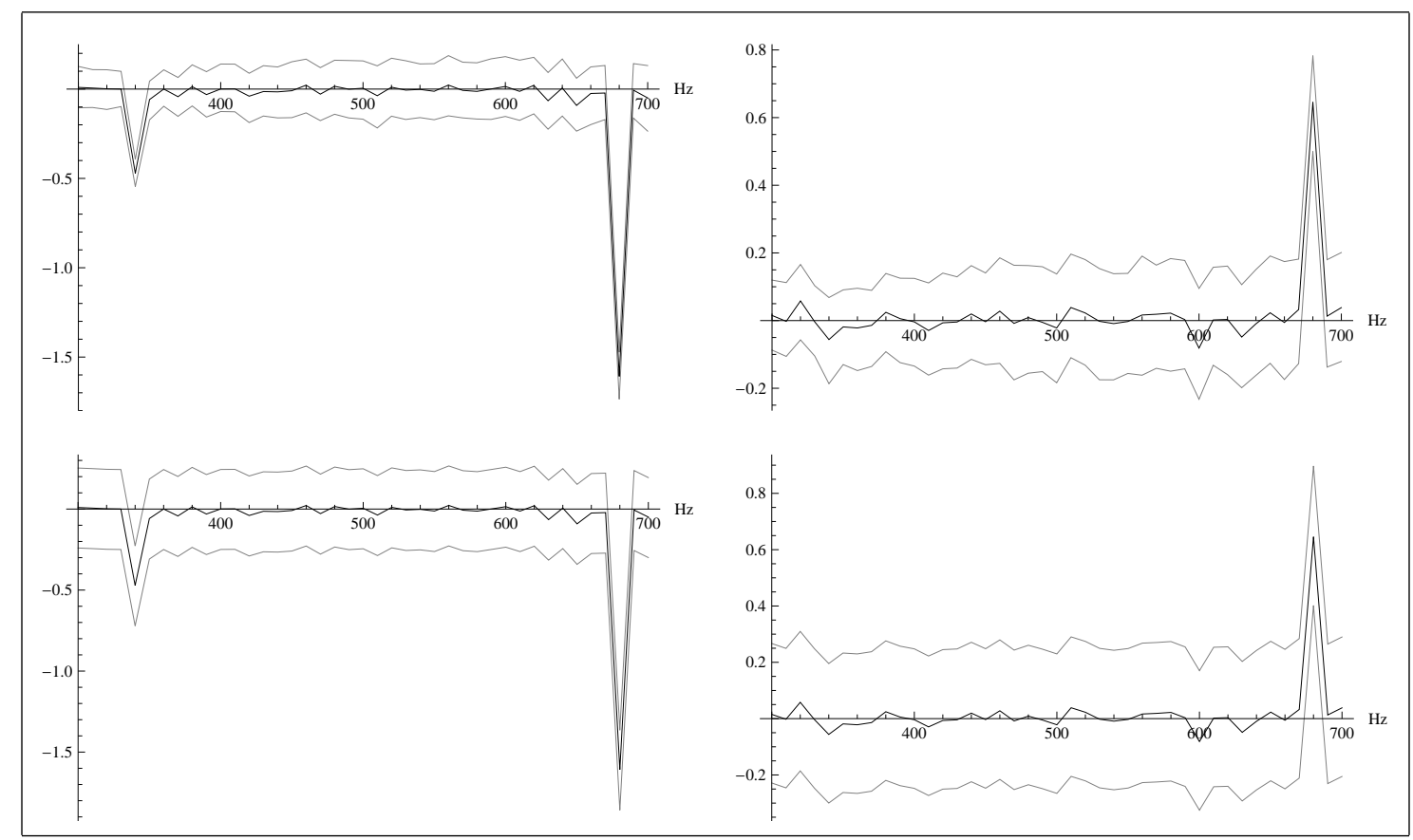

Figure 11: Grey color: pointwise (first row) and simultaneous (second row) confidence intervals for $\operatorname{Re}(a(\lambda, 0))($ left column) and $\operatorname{Im}(a(\lambda, 0))$ (right column), $\lambda \in\{300 \mathrm{~Hz}, 310 \mathrm{~Hz}, \ldots, 700 \mathrm{~Hz}\}$ and $b=30$. Black color: estimators of $R e(a(\lambda, 0))$ and $\operatorname{Im}(a(\lambda, 0))$. Nominal coverage probability is $95 \%$.

[28] P.D. McFadden, Detecting fatigue cracks in gears by amplitude and phase demodulation of the meshing vibration, J. Vib. Acoust. Stress Reliab. Des. 108(2) (1986) 165-170.

[29] C. Capdessus, Aide au diagnostic des machines tournantes par traitement de signal, PH.D dissertation, Institut National Polytechnique de Grenoble, France, 1992.

[30] C. Capdessus, M. Sidahmed, Analyse des vibrations d'un engrenage: cepstre, corrélation spectre, Traitement du Signal 8(5) (1992) 365-372.

[31] M. El Badaoui, New applications of the real cesptrum to gear signals, including definition of a robust fault indicator, Mechanical systems and Signal processing 18 (2004) 1031-1046.

[32] M.El Badaoui, F. Guillet, J. Danière, New applications of the real cepstrum to gear signals, including definition of a robust fault indicator, Mechanical Systems and Signal Processing, 18 (2004) 10311046 . 


\section{Acknowledgements}

The authors gratefully acknowledge the support of CETIM (Centre des Etudes Techniques des Industries Mécaniques de Senlis), which provided the experimental results. Anna Dudek has received funding from the European Union's Horizon 2020 research and innovation programme under the Marie SkłodowskaCurie Grant Agreement No. 655394.
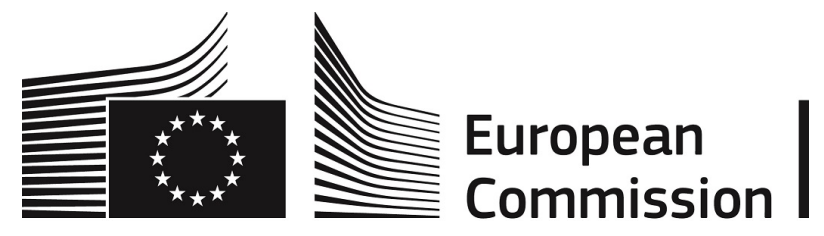